\title{
The Cessation of Continuous Turbulence as Precursor of the Very Stable Nocturnal Boundary Layer
}

\author{
B. J. H. VAN DE WIEL \\ Fluid Dynamics Laboratory, Eindhoven University of Technology, Eindhoven, Netherlands \\ A. F. MOENE \\ Department of Meteorology and Air Quality, Wageningen University, Wageningen, Netherlands \\ H. J. J. JONKER \\ Applied Physics, Delft University of Technology, Delft, Netherlands
}

(Manuscript received 23 February 2012, in final form 7 August 2012)

\begin{abstract}
The mechanism behind the collapse of turbulence in the evening as a precursor to the onset of the very stable boundary layer is investigated. To this end a cooled, pressure-driven flow is investigated by means of a local similarity model. Simulations reveal a temporary collapse of turbulence whenever the surface heat extraction, expressed in its nondimensional form $h / L$, exceeds a critical value. As any temporary reduction of turbulent friction is followed by flow acceleration, the long-term state is unconditionally turbulent. In contrast, the temporary cessation of turbulence, which may actually last for several hours in the nocturnal boundary layer, can be understood from the fact that the time scale for boundary layer diffusion is much smaller than the time scale for flow acceleration. This limits the available momentum that can be used for downward heat transport. In case the surface heat extraction exceeds the so-called maximum sustainable heat flux (MSHF), the near-surface inversion rapidly increases. Finally, turbulent activity is largely suppressed by the intense density stratification that supports the emergence of a different, calmer boundary layer regime.
\end{abstract}

\section{Introduction}

For many purposes related to meteorology and climate, a proper understanding of the boundary layer processes in stably stratified conditions is essential (Beljaars and Viterbo 1998). Despite its importance, a general framework of the stable boundary layer (SBL) is still lacking (Derbyshire 1999b; Fernando and Weil, 2010; Baklanov et al. 2011). Nevertheless, it appears useful to classify stable boundary layers into two major prototypes (Mahrt et al. 1998): the weakly stable boundary layer (WSBL) and the very stable boundary layer (VSBL).

Weakly stable boundary layers are characterized by the presence of continuous turbulence and tend to occur

Corresponding author address: B. J. H. Van de Wiel, Fluid Dynamics Laboratory, Eindhoven University of Technology, P.O. Box 513, 5600 MB Eindhoven, Netherlands.

E-mail: b.j.h.v.d.wiel@tue.nl in windy and/or cloudy conditions. This type of boundary layer is rather well understood, and both fluxes and profiles more or less obey local similarity scaling as formulated by Nieuwstadt (1984) and Derbyshire (1990). Therefore, for most practical modeling applications, our representations of the WSBL are acceptable to some degree (Sorbjan 2006; Steeneveld et al. 2006; Beare et al. 2006; Cuxart et al. 2006; Svensson et al. 2011).

In contrast, very stable boundary layers are poorly understood. This type of boundary layer is characterized by turbulence that is weak or virtually absent [in the sense described by Zilitinkevich et al. (2008)]. In the latter case, we may speak of a so-called radiative SBL because in the absence of vigorous turbulent heat transport, clear-air radiation is the dominant thermodynamic processes (Van de Wiel et al. 2003). Recently, considerable effort has been made to understand various processes within such VSBLs, such as longwave radiative cooling (Sun et al. 2003b; Edwards 2009), subtle turbulent activity on the small and mesoscale (Mahrt 
2011; Mahrt et al. 2012), low-level jet formation (Banta 2008), and katabatic effects over sloping terrain (e.g., Shapiro and Fedorovich 2008). Apart from this relatively calm "background behavior," disturbances may suddenly appear in the form of infrequent turbulent mixing events of various origin (Poulos et al. 2002; Sun et al. 2003a).

Although classification into WSBL and VSBL seems attractive as a qualitative concept, a formal criterion for existence of different SBL regimes does not exist. Therefore, for given external forcings, it is a priori unknown whether a certain night will manifest itself as being weakly or very stable. The aim of the present work is to predict (in terms of external forcings) the critical point where turbulence cannot maintain its vigorous continuous character. In view of the definitions above, this point will then determine the onset of the VSBL.

At night stabilizing radiative cooling of the surface is competing with wind shear that tries to generate turbulence mixing. The relative magnitude of those processes will determine the presence or absence of turbulence. In turn, those processes are externally driven by the incoming longwave radiation at the top of the boundary layer (i.e., contribution or absence of low-level clouds) and by the synoptic pressure gradient (Van de Wiel et al. 2002b; Svensson et al. 2011). The impact of this forcing mechanism is illustrated in Fig. 1. From a 10-yr dataset at Cabauw, the Netherlands [Royal Netherlands Meteorological Institute (KNMI)], clear nights (net radiation $<-20 \mathrm{~W} \mathrm{~m}^{-2}$ ) were selected and subdivided into four classes of geostrophic wind. To ensure reasonable stationarity, nights with more than $5 \mathrm{~m} \mathrm{~s}^{-1}$ variation in the geostrophic wind between 1200 and 0000 UTC were not considered. The geostrophic wind was obtained by analyzing surface pressure observations from eight synoptic stations in a radius of $75 \mathrm{~km}$ around Cabauw (F. C. Bosveld 2012, personal communication). As clear nights have similar radiative forcing, the magnitude of the geostrophic wind is expected to be decisive on the existence of turbulence. The temporal evolution of the kinematic turbulent stress in the evening given in Fig. 1 reveals this sensitivity. In general, turbulent stresses decrease in the late afternoon due to the decay of convection. Additionally, the stress response reveals an onoff character during nighttime: turbulent activity tends to become negligibly small for geostrophic winds below $3-6 \mathrm{~m} \mathrm{~s}^{-1}$.

This finding seems to be in accordance with Cabauw observations by Nieuwstadt (1984), who restricted his analysis to geowinds above $5 \mathrm{~m} \mathrm{~s}^{-1}$ in order to ensure continuous turbulence. In his study on boundary layer decoupling, Derbyshire (1999a) shows an instructive example for two nights at Cardington observational station

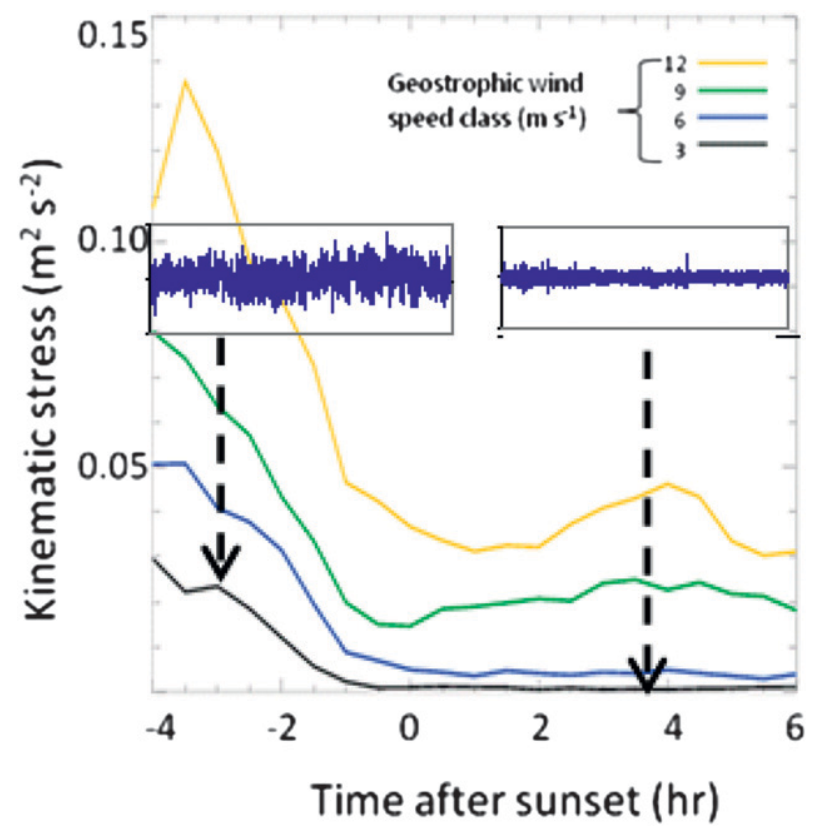

FIG. 1. Time evolution of kinematic stress at the KNMI Cabauw observatory (5-m level). The curves represent median stress levels for four geostrophic wind classes $\left( \pm 1 \mathrm{~m} \mathrm{~s}^{-1}\right)$, as indicated in the graph. Typical vertical velocity fluctuations $(10 \mathrm{~Hz})$ are indicated as an illustration of strongly and weakly turbulent periods.

(in Cardington, United Kingdom) with similar net radiative cooling $\left(\sim 60 \mathrm{~W} \mathrm{~m}^{-2}\right)$. The night with weaker geostrophic forcing (estimated $\sim 6 \mathrm{~m} \mathrm{~s}^{-1}$ ) corresponds to a turbulent heat flux close to zero, whereas in the case of stronger geostrophic wind $\left(\sim 10 \mathrm{~m} \mathrm{~s}^{-1}\right)$ this flux is about $-25 \mathrm{~W} \mathrm{~m}^{-2}$, on average. In the context of largeeddy simulation, sudden regime transitions in response to changing geostrophic forcing and surface cooling have been reported by Jiménez and Cuxart (2005). Likewise, Acevedo et al. (2012) observed a transition to the VSBL whenever the geostrophic wind was below 5-7 $\mathrm{m} \mathrm{s}^{-1}$, in a study on idealized SBL systems with various types of turbulence closure. In accordance with this, two distinct SBL regimes where found by Estournel and Guedalia (1985), using a one-dimensional turbulent kinetic energy (TKE) model with a radiative transfer code. Finally, qualitatively similar results were reported in wind tunnel experiments by Ohya et al. (1997).

In general fluid mechanics, transitions from laminar to turbulent flow regimes have been studied extensively (Kundu and Cohen 2008). To this end the hydrodynamic stability of exact analytical solutions to the Navier-Stokes equations in case of laminar flow are studied by imposing small-amplitude wavelike perturbations on those solutions. Under certain conditions some of these "modes" may grow in time and hence point at the onset of turbulence. A well-known example 
relevant for stratified flows is given in Miles (1961) and Howard (1961). Unfortunately, such rigorous analysis is not viable for our reverse problem (Zilitinkevich et al. 2007; Mauritsen and Svensson 2007): no general analytical solutions for turbulent flows are available. This means that a well-defined description of the basic state is lacking, so that hydrodynamic stability analysis seems impossible.

With respect to nocturnal boundary layers, an interesting view was introduced by Revelle (1993) and adopted by, for example, McNider et al. (1995), Derbyshire (1999a), Van de Wiel et al. (2002a), and Acevedo et al. (2012). Rather than analyzing the exact problem, they studied regime transitions in parameterized systems. This revealed intriguing nonlinear behavior of the SBL, including bifurcations, oscillations, decoupling, and collapse of turbulence. In particular, the pioneering study on a truncated set of equations by McNider et al. (1995) already showed the potential existence of both "warm" and "cold" nocturnal boundary layer solutions in response to varying geostrophic wind forcing. In addition, an analytical stability analysis on idealized (linear) wind and temperature profiles was performed by Derbyshire (1999a). This result was important, in a sense that it formally proved that the collapse of turbulence arises from true physical feedbacks rather than from numerical artifacts.

In Van de Wiel et al. (2007, hereafter VDW07), Derbyshire's analysis was extended, in a sense that more realistic Monin-Obukhov type of profiles were considered. To this end a Couette flow forced by a moving plate at the top was studied. It was proven that instability in such "fixed shear" system results from the fact that the downward heat flux in a stratified flow is limited to a maximum: the heat flux vanishes for very small [low Richardson number (Ri)] and very large temperature gradient (high $\mathrm{Ri}$ ). The mechanism is evident: if the extraction of heat at the surface is larger than the maximum that can be supplied by the flow, then it will lead to a rapid increase of the near-surface inversion. In turn, this enforces the reduction of mixing efficiency so that the heat supply decreases further. Eventually, virtually "all" turbulent activity will be suppressed by the intense density stratification. Section 2 will briefly outline this maximum sustainable heat flux (MSHF) theory. At this point it is important to note that true laminar flows-in a strict sense-are unlikely to occur in geophysical systems (Galperin et al. 2007). More likely, as pointed out by Mahrt and Vickers (2006), Mauritsen and Svensson (2007), Zilitinkevich et al. (2008), Sorbjan (2010) and Grachev et al. (2012), this new, cold atmospheric regime will still show some remaining turbulence of weak and discontinuous character.
Apart from dynamical consequences for Couette flows, the existence of the maximum in atmospheric stable boundary layers has been reported in many observational studies (e.g., De Bruin 1994; Malhi 1995; Mahrt et al. 1998; Basu et al. 2006; Grachev et al. 2005). Already in 1971 it was shown by Taylor that its existence simply follows mathematically from the existence of nondimensional, log-linearly shaped wind and temperature profiles. In fact, any realistic similarity function will support vanishing fluxes at neutral and high stability, and thus imply the existence of such maximum (e.g. Basu et al. 2008; Grachev et al. 2005).

Unfortunately, only for the specific Couette flow can the collapse of turbulence be directly explained by this MSHF. This is due to the assumption of fixed bulk shear, which is crucial in deriving the MSHF (for infinite shear, the heat flux increases monotonically with increasing temperature gradient). In reality such fixed shear cannot exist; atmospheric flows are forced by pressure gradients rather than by a moving plate, and pressure forces are balanced by turbulence stresses. When turbulent activity ceases in response to cooling, frictional forces become weak as well. Consequently, the net force accelerates the flow, which increases the ambient shear, so that finally the boundary layer is able to become turbulent again (Businger 1973; Derbyshire 1999a).

So, what then causes the (temporary) collapse of turbulence in such flows? In order to understand this, the dynamics of a simplified system is studied in analogy to the atmospheric case. A pressure-driven channel flow is investigated by using a local similarity model. Surface cooling is applied externally by prescribing the heat extraction at the surface. The configuration follows the setup of Nieuwstadt (2005), Moene et al. (2010), and Flores and Riley (2011). Those studies investigated the collapse of turbulence in response to increased surface cooling at constant pressure gradient, by solving the Navier-Stokes equations directly on a computational grid [so-called direct numerical simulation (DNS)]. They found a total collapse whenever the cooling exceeded a certain critical value. Apart from the Reynolds number, the overall effect of the external forcings was combined into a single nondimensional parameter $h / L_{\text {ext }}$ (section 3). Within this nondimensional framework, the "fixed pressure-increased cooling" configuration is equivalent to the "variable pressure gradient-fixed cooling (in terms of net radiation)" case of Fig. 1. We use this equivalence to extend the results to actually observed atmospheric cases in the companion paper (Van de Wiel et al. 2012).

The present analysis confirms that pressure-driven accelerations invalidate an assumption of fixed shear in the long term. As such, the flow behavior is unconditionally 
turbulent in the long term. In contrast, the period after the onset of cooling reveals largely different behavior. In this time window, diffusion processes turn out to be much faster than the typical acceleration time scale [in the atmosphere, the latter is typically of order $f^{-1}$, that is, of several hours, whereas diffusion may take place on a time scale $O(10 \mathrm{~min})$; see section 5]. The slow acceleration effectively limits the total momentum in the lower atmosphere. In turn, this implies a limitation on the downward heat transport in a similar way as in the Couette case. Finally, this analogy enables both quantitative prediction and interpretation of the collapse phenomenon in terms of the MSHF theory.

The paper is organized as follows: In the next section, the MSHF hypothesis for Couette flows is briefly summarized. In section 3 a stably stratified channel flow is studied by utilizing a local similarity model. Numerical results are explained by analytical analysis in section 4 , and the framework is validated in section 5. Finally, atmospheric implications and conclusions are given in sections 6 and 7, respectively.

\section{Background: The maximum sustainable heat flux in a Couette flow}

The point of departure is the analysis of VDW07, where it is shown that the downward heat flux in case of stably stratified Couette flow is limited to a certain maximum: "the maximum sustainable heat flux." A Couette flow is driven by a moving plate at the upper boundary. The results by VDW07 are briefly summarized.

The Couette flow is presented in Fig. 2. In its steady state, momentum and heat fluxes become constant with height and the general description in terms of local properties (Nieuwstadt 1984) degenerates to simple Monin-Obukhov similarity (Monin-Obukhov 1954) using surface fluxes only. Next, fluxes are expressed in terms of discrete differences of wind and temperature by integration over the domain depth. We restrict ourselves to the most simple similarity functions: $\phi_{m, h}=1+\alpha z / L$. However, it is shown below that the existence of a heat flux maximum merely emerges from the fact that mixing efficiency decreases with stability. Because this characteristic is a general feature in observationally based similarity functions, the qualitative results are not expected to be very sensitive to the specific choice of $\phi_{m, h}$ (Delage et al. 2002; Basu et al. 2008). Integration between $z=z_{0}$ and $z=h$ relates the normalized fluxes to the wind and temperature differences $\Delta U$ and $\Delta \theta$ over the domain depth as shown:

$$
\frac{u_{*_{0}}^{2}}{(\Delta U)^{2}} \frac{\left[\ln \left(h / z_{0}\right)\right]^{2}}{\kappa^{2}}=\left(1-\alpha R_{b}\right)^{2}
$$

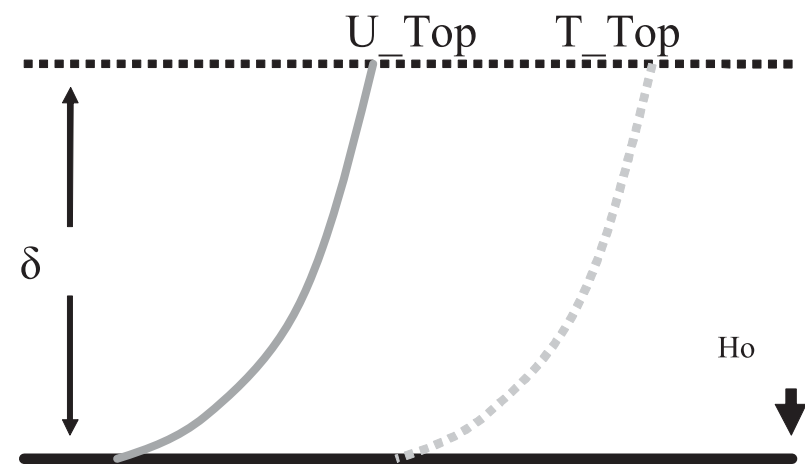

FIG. 2. Schematic picture of Couette flow configuration, as defined in VDW07.

$$
\frac{u_{* 0} \theta_{* 0}}{(\Delta U)^{3}} \alpha h \frac{g}{\theta_{\text {ref }}} \frac{\left[\ln \left(h / z_{0}\right)\right]^{2}}{\kappa^{2}}=\alpha R_{b}\left(1-\alpha R_{b}\right)^{2},
$$

with the bulk Richardson number $R_{b} \equiv \Delta \theta\left(h g / \theta_{\text {ref }}\right) /$ $(\Delta U)^{2}$. It is important to note that, although mathematically the choice of this simple $\phi_{m, h}$ shape asymptotically implies a "critical" value of the bulk Richardson number equal to $1 / \alpha$, it has no direct physical meaning for the collapse mechanism as such. Indeed, the existence of a "critical" Richardson number for the transition from turbulence to laminar can be questioned, particularly in geophysical flows (Galperin et al. 2007). More likely, some weak turbulence remains after "collapse" (Maurtisen and Svensson 2007; Zilitinkevich et al. 2008; Sorbjan 2010, Grachev et al. 2012). However, as mentioned above, more complex $\phi_{m, h}$, which account for this residual turbulence (e.g., Beljaars and Holtslag 1991), allow a similar transition mechanism from a strong to a weakly turbulent regime.

Consider Eq. (2) in case of fixed $\Delta U$. The imposed $H_{0}$, with $H_{0}=\rho c_{p} u *_{0} \theta *_{0}$, corresponds to certain value(s) of $\Delta \theta$. Alternatively, we could have fixed $\Delta \theta$ and have diagnosed $H_{0}$. Mathematically, $H_{0}$ depends in a cubic way on $R_{b}$, hence on $\Delta \theta$. In a physical sense, $\left|H_{0}\right|$ may decrease by two causes:

in the neutral limit, $\left|H_{0}\right|$ decreases with decreasing $\Delta \theta$ (gradient limited transport); and

in the very stable limit, $\left|H_{0}\right|$ decreases with increasing $\Delta \theta$ (mixing limited transport).

Those effects lead to a well-defined flux maximum when plot as a function of $\Delta \theta$ or $R_{b}$ (Fig. 3a; Taylor 1971; Malhi 1995). Clearly, the flow will be unable to support a surface heat extraction that exceeds this maximum: the inversion rapidly intensifies, which further suppresses vertical turbulent transport, until turbulence finally is unable to survive the intense density stratification. This results in a different flow regime with highly suppressed 

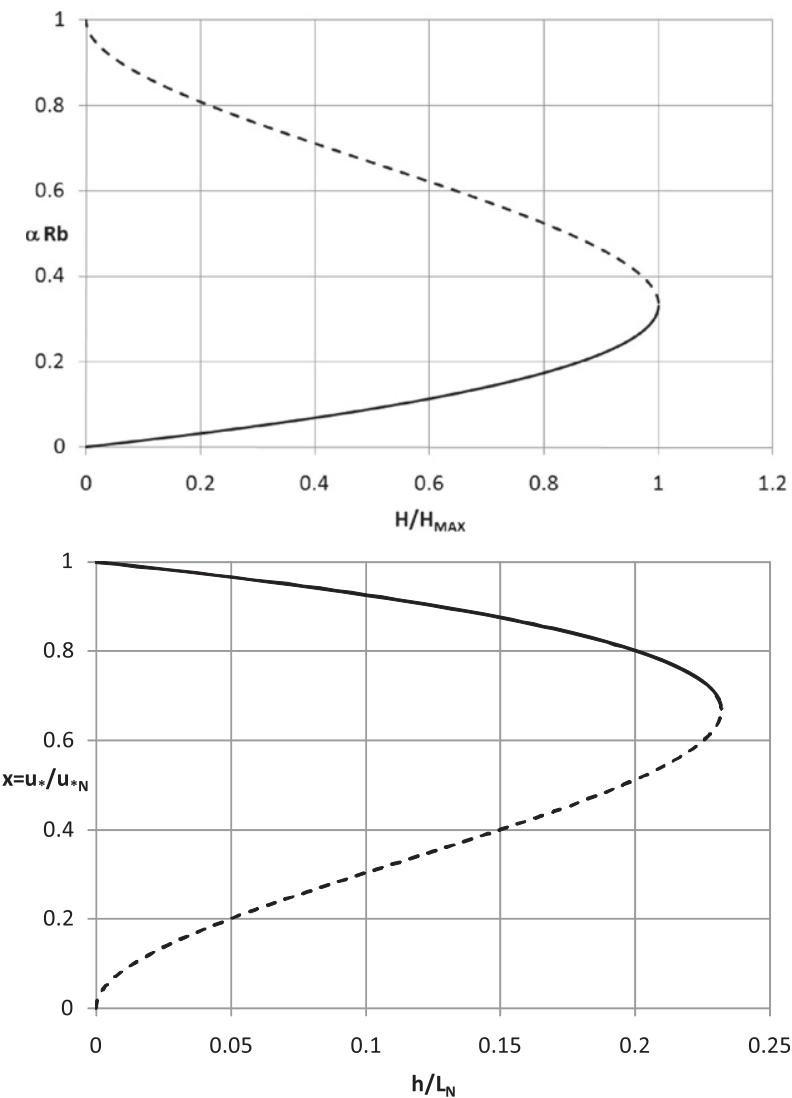

FIG. 3. (top) Solution curve relating the bulk Richardson number and the normalized heat flux. The maximum flux is found at $\alpha R_{b}=1 / 3$. The part of the solution unstable to perturbations is indicated by the dashed line (see text). (bottom) Solution curve in terms of normalized friction velocity and stability parameter $h / L_{N}$, Eq. (6). Note that the bottom panel is simply a mirrored, rescaled version of the top panel (see text).

turbulence. In Eq. (2) $d H_{0} / d R_{b}=0$ occurs at $\alpha R_{b}=1 / 3$, and the expression for the MSHF becomes

$$
H_{\mathrm{MAX}}=\frac{4}{27} \rho c_{P} \frac{\kappa^{2}}{\left[\ln \left(h / z_{0}\right)\right]^{2}} \frac{\theta_{\text {ref }}}{\alpha h g}(\Delta U)^{3} .
$$

Note that this is an actual limit for strong, continuous turbulence in stratified Couette flows. One should not confuse this actual MSHF with the potential MSHF defined by Derbyshire (1990). His useful definition of the maximum sustainable heat flux sets an absolute upper limit based on energy considerations (Derbyshire 1990). The latter represents the amount of heat flux that would be generated if all the work by the flow could be used to mix heat downward (i.e., if mixing were not hampered by stratification itself, as here). Roughly speaking, this implies multiplication of the total shear production with the "critical" Richardson number (implying $R_{b} \approx 1 / \alpha$ ). However, before this energy constraint is reached, exceedance of the actual limit already causes a collapse of turbulence. As shown, such transition typically occurs at lower bulk Richardson numbers $\left[R_{b} \approx(1 / 3)(1 / \alpha)\right]$.

For completeness we note that mathematically stability analysis (VDW07) indicates that the upper part of the solution curve is unstable to perturbations. For brevity reasons this aspect is not discussed here. Although the graphical representation in Fig. 3a appeals for direct physical interpretation, a different but mathematically fully equivalent representation is needed in order to relate this Couette flow result to our channel flow analysis at a later stage (section 5; Fig. 10). Readers not interested in this may skip this part and continue with section 3 .

In pressure-driven flows, $\Delta U$ is a not a natural scaling parameter as it is a priori unknown, unlike in the Couette case. Instead, the equilibrium stress at the surface is known from the force balance. This defines the equilibrium friction velocity $u_{* \mathrm{EXT}}$ as a natural scaling parameter (section 3). Next, we can modify the Couette scaling parameter $\Delta U$ in such a way that it resembles a friction velocity, so that both flows can be compared. Hereto we define the "neutral friction velocity" $u *_{N}$ [following Holtslag and Van Ulden (1982)] as

$$
u_{* N} \equiv \Delta U \frac{\kappa}{\ln \left(h / z_{0}\right)} .
$$

It is the friction velocity that a system would have under neutral conditions with given $\Delta U$. Next, we normalize the surface heat flux $H_{0}$ with $u_{*_{N}}^{3}$ by defining $h / L_{N}$, with the modified Obukhov length $L_{N}$ being

$$
L_{N}=\frac{u_{*_{N}}^{3}}{H_{0} / \rho c_{p}} \frac{T_{\mathrm{ref}}}{\kappa g} .
$$

Also the vertical axis $\alpha R_{b}$ is modified. It is related to the friction velocity by using $1-\alpha R_{b}=u *_{0} / u *_{N}$ [Eq. (1)]. The vertical axis is "mirrored," in a sense that neutral cases now appear in the upper part. Figure 3a is now mathematically "mapped" onto Fig. 3b, which will be compared in the remaining sections. Figure $3 \mathrm{~b}$ can be represented by Eq. (6), which can be derived from Eqs. (1) and (2), and will be used later. Equation (6) expresses $u_{*} / u_{*_{N}}$ (denoted as $x$ ) in terms of $h / L_{N}$ as shown:

$$
\kappa \frac{\Delta U}{u_{* N}}=x\left[\ln \left(\frac{h}{z_{0}}\right)+\alpha \frac{1}{x^{3}} \frac{h}{L_{N}}\right]
$$




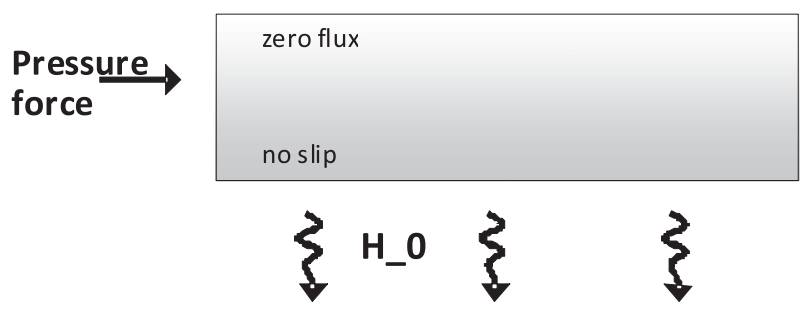

FIG. 4. Schematic picture of the channel flow configuration with the pressure gradient force and prescribed surface heat extraction as external parameters. Decreasing temperature is indicated by increasing gray scale.

\section{Collapse of turbulence in a stably stratified channel flow}

\section{a. Flow configuration and model setup}

The setup is inspired by Nieuwstadt (2005), who studied a stably stratified channel flow in analogy to nocturnal boundary layers (Fig. 4). To this end the incompressible Navier-Stokes equations under the Boussinesq assumption were solved numerically in a $3 \mathrm{D}$ grid configuration. As no turbulence model is involved, he refers to those simulations as DNS. The study reveals that turbulence is unable to survive for large cooling rates. Here, we aim to understand the instability mechanism behind this collapse of turbulence, by utilizing a simple model based on local similarity theory. The advantage of such a "surrogate model" is that it can be analyzed analytically. Also, the closure resembles the type of parameterizations that are common in numerical weather prediction models. In a previous study by the present authors (Moene et al. 2010), an extensive comparison between the simple model and DNS has been carried out. With both models laminarization occurred at large cooling rates. Furthermore, close agreement was found with respect to the mean and the flux profiles in the laminar and the turbulent flow regimes. Inspired by this, Monin-Obukhov similarity functions were diagnosed directly from the 3D flow simulations (Van de Wiel et al. 2008). It revealed that the dimensionless shear and temperature gradients are quite well described by the log-linear similarity function that is used in the present study: $\phi_{M, H}=1+\alpha z / \Lambda$ (with $\alpha=4-5$, and $\Lambda$ is the local Obukhov length as defined in appendix A).

In the aforementioned studies, a frictional Reynolds number $\mathrm{Re}_{*}=u_{*_{\mathrm{EXT}}} h / \nu$ equal to 360 was assumed (see below). This corresponds to a bulk Reynolds number $O(7000)$, that is, small compared to typical atmospheric values. In Moene et al. (2010) this low value was chosen in order to allow direct comparison with DNS. Here, however, we exclude the dynamic effects of molecular viscosity, in order to allow direct comparison with atmospheric flows. Unlike in Van de Wiel et al. (2008) and Moene et al. (2010), where both turbulent and molecular contributions to diffusion were added, we set the molecular contributions to zero. As we model an aerodynamic "smooth" flow, viscosity only enters the system via the effective roughness of the flow, which is taken as $z_{0} / h=1 /\left(7 \mathrm{Re}_{*}\right)$ (see below). As this influence is passive/"off-line," the actually simulated nondimensional profiles will have "no knowledge" of the flow Reynolds number. As such the scaled temperature and wind profiles are representative for rough, high Reynolds number flows as well. Therefore, without loss of generality we take $\mathrm{Re}_{*}=360$ for consistency with the studies above.

In order to impose a pressure gradient, the value of $\mathrm{Re}_{*}$ is fixed: $\mathrm{Re}_{*} \equiv u *_{\text {EXT }} h / \nu$, with $u *$ EXT $\equiv$ $\sqrt{-(1 / \rho)(\partial P / \partial x) h}$, and $h$ is the channel height. In steady state $u *$ EXT equals surface friction velocity $u_{* 0}$ [Eq. (8)].

- The surface heat flux is prescribed by imposing the external stability parameter $h / L_{\mathrm{EXT}}$, defined as

$$
h / L_{\mathrm{EXT}}=\frac{\kappa g h}{T_{\mathrm{ref}} \rho c_{p}} \cdot \frac{H_{0}}{u_{* \mathrm{EXT}}^{3}} .
$$

As such, our system is governed by two external parameters: $\mathrm{Re}_{*}$ and $h / L_{\mathrm{EXT}}$. A zero-flux boundary condition for momentum and heat is applied at the top, and we assume no slip at the height $z=z_{0}$. The conservation equations read

$$
\begin{aligned}
& \frac{\partial U}{\partial t}=-\frac{1}{\rho} \frac{\partial P}{\partial x}+\frac{1}{\rho} \frac{\partial \tau}{\partial z} \\
& \frac{\partial T}{\partial t}=-\frac{1}{\rho c_{p}} \frac{\partial H}{\partial z} .
\end{aligned}
$$

First-order closure is adopted to parameterize the local stress $\tau$ and heat flux $H$ as follows:

$$
\begin{aligned}
\frac{\tau}{\rho} & =(\kappa z)^{2}(\partial U / \partial z) f(\mathrm{Ri}) \frac{\partial U}{\partial z} \\
\frac{H}{\rho c_{p}} & =(\kappa z)^{2}(\partial U / \partial z) f(\mathrm{Ri}) \frac{\partial T}{\partial z}
\end{aligned}
$$

with

$$
\begin{aligned}
& f(\mathrm{Ri})=(1-\alpha \mathrm{Ri})^{2} ; \quad \mathrm{Ri} \leq 1 / \alpha \\
& f(\mathrm{Ri})=0 ; \quad \mathrm{Ri}>1 / \alpha
\end{aligned}
$$

and the gradient Richardson number 


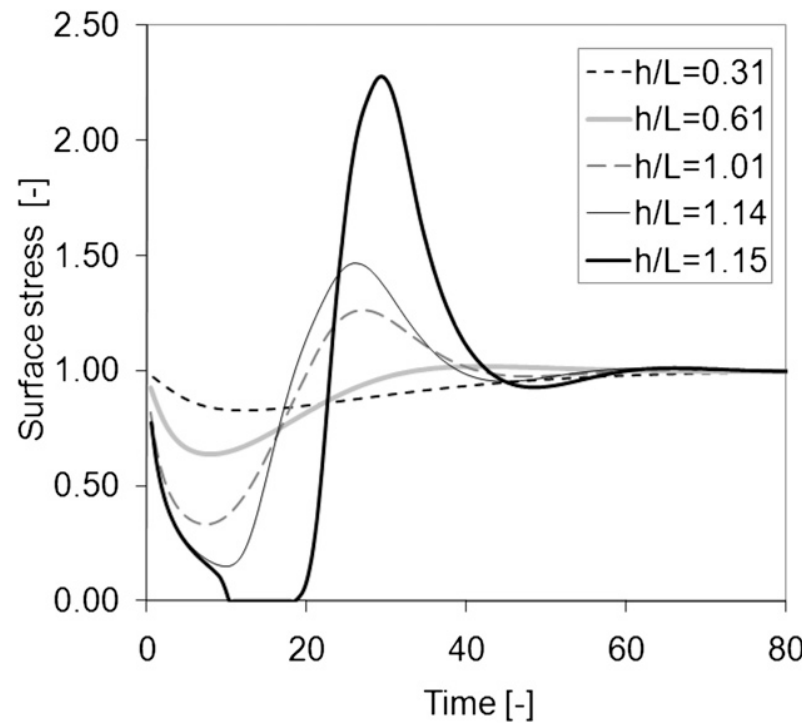

FIG. 5. Normalized surface stress $u_{*_{0}}^{2} / u_{*_{\mathrm{EXT}}}^{2}$ as a function of dimensionless time for different surface cooling rates, represented by the dimensionless parameter $h / L_{\mathrm{EXT}}$.

$$
\mathrm{Ri} \equiv \frac{g}{T_{\text {ref }}} \frac{\partial T / \partial z}{(\partial U / \partial z)^{2}}
$$

The stability functions in Eq. (12) mathematically correspond to the log-linear similarity functions: $\phi_{M, H}=1+$ $\alpha z / \Lambda$ (e.g., England and McNider 1995; King and Connolly 1997). We take $\alpha=5$ as a reasonable representation of atmospheric observational studies (e.g. Högström 1996). Modifications to Van de Wiel et al. (2008) and Moene et al. (2010) are 1) smooth flow modeled analogous to rough flow with an effective roughness length $z_{0} / h=1 /\left(7 \mathrm{Re}_{*}\right)$ (Kundu and Cohen 2008) and 2) a zero-flux upper boundary condition instead of a fixed temperature condition. It was verified that the dynamics of the modified model closely resembled the dynamics of the original model.

\section{$b$. The collapse of turbulence}

Simulations were performed for different cooling rates, all starting from a stationary, neutrally stratified initial flow. Figure 5 shows the evolution of surface stress (scaled by its initial value) as a function of dimensionless time. The normalization time scale used is $t_{*}=\left(h / u_{* \mathrm{EXT}}\right) /$ $\left(h / L_{\mathrm{EXT}}\right)$, based on the characteristic time scale for diffusion (appendix B; cf. also Flores and Riley 2011). For weak cooling the stress response is relatively modest. For stronger cooling, however, the calculated stress reaches smaller values until, for $h / L=1.15$, stresses become zero during a certain period of time. Apparently, continuous turbulence cannot be maintained under strong

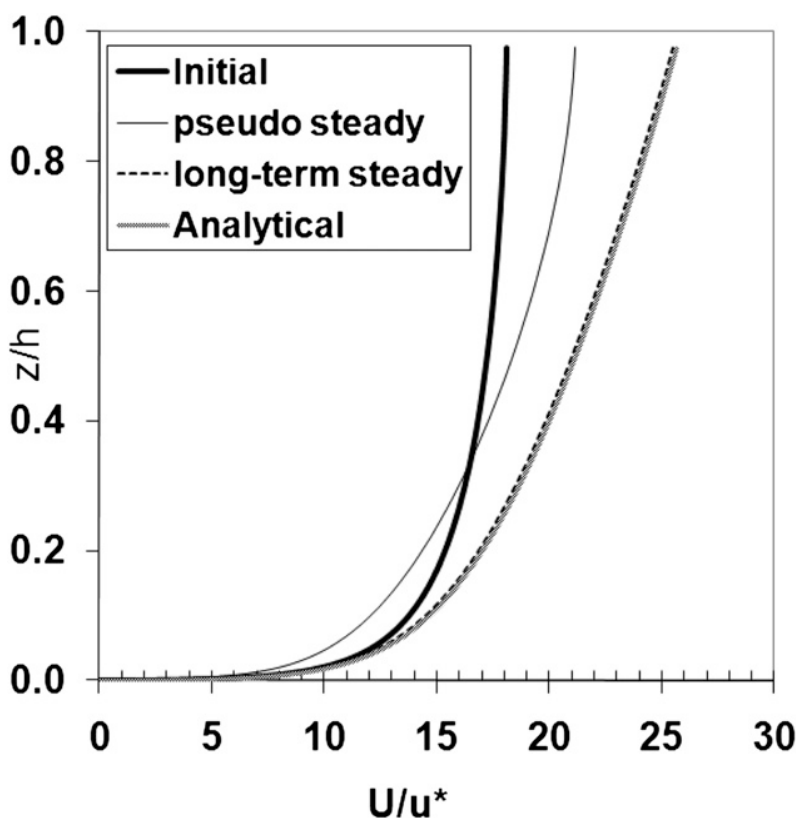

FIG. 6. Simulated wind profile evolution for $h / L_{\mathrm{EXT}}=0.61$. The neutral initial profile, the pseudosteady profile (see section 4), and the long-term steady profile are given. Also, the analytical equilibrium solution (see section 4) is given.

cooling. Basically, the simulation period can be divided in three distinct subperiods or submoments:

- the initial condition (IC) $t / t_{*}=0$;

- a period of minimum stress level, which we will later refer to as the "pseudosteady state" (PSS), $t / t_{*} \approx 5-10$; and - the long-term steady state (SS), $t / t_{*}>60$.

First, the long-term behavior of the simulations is considered. It appears that a turbulent steady state is reached irrespective of the imposed cooling. This has important conceptual implications: in pressure-driven systems, the flow is attracted to a turbulent equilibrium state. The recovery of turbulence is understood from the stability-shear feedback: perturbation of the equilibrium by a sudden increased stability leads to reduced boundary layer friction. This initiates flow acceleration by the pressure gradient until the shear is large enough to restore the turbulent surface stress to its original value. Figure 6 illustrates this acceleration in response to initiated surface cooling. In contrast to the long-term behavior, strongly cooled cases clearly indicate a temporary collapse of turbulence (a period that may last for several hours in the atmosphere; section 5). Below we will explore the underlying mechanism for such collapse.

\section{Theoretical analysis}

In this section a theoretical prediction model for the collapse of turbulence is formulated. First, analytical 
solutions for the long-term steady state are introduced. Hereto, novel equilibrium formulations for wind and temperature profiles are derived that explicitly take into account the vertical divergence of fluxes. Interestingly, the well-known Monin-Obukhov similarity profiles emerge as a logical asymptotic case in the limit $z \downarrow z_{0}$, as shown in appendix A. From this prove such things as a "constant flux layer" cannot formally exist. Hence, one does not need to have a "layer of constant flux" to formulate MO similarity as a useful approximation near the surface. The main goal of the analysis is, however, to pave the path for pseudosteady (PS)-state solutions, because they can be formulated fully analogous to the long-term solutions (section 4b). Finally, it is shown that also in this pseudosteady state, the downward heat supply is limited to a certain maximum as in the previously discussed Couette example.

\section{a. Analytical solutions for the long-term steady state}

Our governing equations read

$$
\frac{\partial U}{\partial t}=-\frac{1}{\rho} \frac{\partial P}{\partial x}+\frac{1}{\rho} \frac{\partial \tau}{\partial z} \quad \text { and } \quad \frac{\partial T}{\partial t}=-\frac{1}{\rho c_{p}} \frac{\partial H}{\partial z},
$$

subject to the following boundary conditions:

$$
\begin{aligned}
& U=0 \quad \text { at } \quad z=z_{0} ; \quad \tau=0 \quad \text { at } \quad z=h \\
& H=H_{0} \quad \text { at } \quad z=z_{0} ; \quad H=0 \quad \text { at } \quad z=h .
\end{aligned}
$$

In equilibrium, the shape of the profiles does not change in time as shown:

$$
0=\frac{\partial(\partial U / \partial z)}{\partial t}=\frac{1}{\rho} \frac{\partial^{2} \tau}{\partial z^{2}} \quad \text { and } \quad 0=\frac{\partial(\partial T / \partial z)}{\partial t}=\frac{1}{\rho} \frac{\partial^{2} H}{\partial z^{2}},
$$

which corresponds to the following linearly decreasing flux profiles:

$$
\tau / \tau_{0}=u_{*}^{2} / u_{* \mathrm{EXT}}^{2}=(1-z / h) \text { and } H / H_{0}=(1-z / h),
$$

where we used $z_{0} \ll h$. The system is closed by relating the local fluxes to the local gradients of wind and temperature, so that an expression for the steady-state wind profile is obtained (using $q \equiv z / h$; Appendix A) as shown:

$$
\begin{aligned}
\kappa \frac{U(q)}{u_{* \mathrm{EXT}}}= & \left(2 \sqrt{1-q}-2 \operatorname{atanh} \sqrt{1-q}+\alpha q \frac{h}{L}\right) \\
& -\left(2 \sqrt{1-q_{0}}-2 \operatorname{atanh} \sqrt{1-q_{0}}+\alpha q_{0} \frac{h}{L}\right) .
\end{aligned}
$$

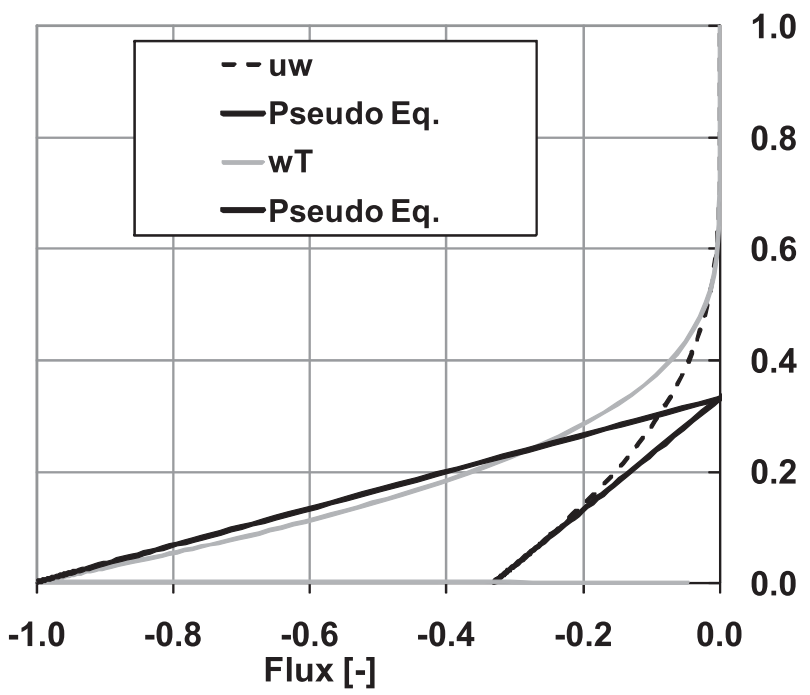

FIG. 7. Momentum and heat flux profiles for the $h / L_{\mathrm{EXT}}=1.01$ case at PSS $t / t_{*}=\hat{t}_{\mathrm{PS}}$. For comparison, idealized linear flux profiles are drawn. The intersection of the idealized profiles with the vertical axis determines the depth of the PS layer.

By taking $h / L=h / L_{\mathrm{EXT}}$, the expression for the long-term steady state is obtained. For comparison with numerical simulations, we refer to the example given in Fig. 6. An analytical description of the initial condition also follows from Eq. (17), by using $h / L=h / L_{\mathrm{INIT}}$ (stability of initial state). In practice, one may use $2 \operatorname{atanh}(y)=\ln [(1+y) /$ $(1-y)]$ for $y<1$; a condition fulfilled by our scaling.

\section{b. The pseudosteady state}

The concept of PSS is introduced here. First, "pseudosteady time" $t / t_{*}=\hat{t}_{\mathrm{PS}}$ is defined as the time when the level of minimum surface stress is reached (Fig. 5). To appreciate the concept, Fig. 7 shows typical flux profiles at $t / t_{*}=\hat{t}_{\mathrm{PS}}$ as a function of normalized height for $h / L_{\mathrm{EXT}}=1.01$. Note that in this example, we choose $h / L_{\text {EXT }}$ to be larger than in the previous example of Fig. 6 for reasons of clarity: the more strongly cooled case emphasizes the decoupling between the lower and upper domains as explained below. In Fig. 7, the profiles tend to become linear with height as if it were a true steady state [Eq. (16)], but now on a reduced domain extending from $q_{0} \leq q \leq \gamma$ instead of $q_{0} \leq q \leq 1$. This implies that wind and temperature gradients behave "stationary" in this region. As this "stationarity" only can exist for a limited amount of time, it is referred to as the pseudosteady state.

The flux-profile analogy with the long-term steady state is used in order to find the velocity profile during PSS. We use the fact that in PSS the slope of the dimensionless stress profile is -1 (Fig. 7), so that the unknown height of the turbulent layer $\gamma$ is equal to the 
unknown normalized surface stress: $\gamma=\left(u_{* 0 \mathrm{PS}} / u_{*} \mathrm{EXT}\right)^{2}$, with $u_{* 0 \text { PS }}^{2}$ the surface stress during pseudoequilibrium. The profile in the region $q_{0} \leq q \leq \gamma$ is now defined analogous to Eq. (17) as shown:

$$
\begin{aligned}
\kappa \frac{U\left(q, \hat{t}_{\mathrm{PS}}\right)}{u_{* \mathrm{EXT}}}= & x\left(2 \sqrt{1-\frac{q}{x^{2}}}-2 \operatorname{atanh} \sqrt{1-\frac{q}{x^{2}}}\right. \\
& \left.+\alpha \frac{q}{x^{3}} \frac{h}{L_{\mathrm{EXT}}}\right)-x\left(2 \sqrt{1-\frac{q_{0}}{x^{2}}}\right. \\
& \left.-2 \operatorname{atanh} \sqrt{1-\frac{q_{0}}{x^{2}}}+\alpha \frac{q_{0}}{x^{3}} \frac{h}{L_{\mathrm{EXT}}}\right)
\end{aligned}
$$

with $x^{2} \equiv \gamma$. Conceptually, we could have used $\sqrt{1-q / \gamma}$ in Eq. (18) in analogy with $\sqrt{1-q}$ in Eq. (17). Here, however, we wish to emphasize that the surface friction velocity $x$ is the unknown parameter. Equation (18) still contains two unknown parameters: $U\left(\hat{t}_{\mathrm{PS}}, q\right)$ and $x$. This is an important difference from Eq. (17), where a priori knowledge on surface stress is available. Therefore, an additional constraint is needed. An in-depth analysis given in appendix B shows that such constraint appears naturally from the fact that the time scale $\tau_{\text {diff }}$ for diffusion, which builds the PS layer in accordance with the new surface boundary condition, is much smaller than the acceleration time scale $\tau_{\text {acc }}$, which determines the typical lapse time until long-term steady state. This separation of time scales implies that the PSS sets in rapidly. Therefore, in the pseudosteady layer there is insufficient time for significant momentum change, that is,

$$
\int_{q_{0}}^{\gamma}\left[\hat{U}\left(q, \hat{t}_{\mathrm{PS}}\right)-\hat{U}(q, 0)\right] d q \approx 0,
$$

with $\hat{U}\left(\hat{t}_{\mathrm{PS}}, q\right)=U\left(\hat{t}_{\mathrm{PS}}, q\right) / u_{* \mathrm{EXT}}$. According to Eq. (19) changes in the wind profile during $0 \leq \hat{t} \leq \hat{t}_{\mathrm{PS}}$ are mainly due to the diffusive redistribution of momentum and not to a net change of the total momentum. In Eq. (19), $\hat{U}\left(\hat{t}_{\mathrm{PS}}, q\right)$ is provided by Eq. (18) and $\hat{U}(\hat{t}=0, q)$ by Eq. (17) (with $h / L=0$ ), so that a closed set of equations remains with three unknowns, $\hat{U}(\hat{t}=0, q), \hat{U}\left(\hat{t}_{\mathrm{PS}}, q\right)$, and $x$ (or $\gamma$ ). It describes the state at $\hat{t}=\hat{t}_{\mathrm{PS}}$ to which the system tries to evolve in response to instantaneous surface cooling.

\section{c. Physical interpretation: The velocity crossing point}

Equation (19) is important from a mathematical perspective, in a sense that it closes the set of equations. But, it has even more relevance with respect to the physical cause of instability. Figure 8 represents the evolution of the velocity profile toward PSS for the case introduced in Fig. 6. Indeed, it occurs that momentum tends to be more or less conserved over depth $\gamma$. Interestingly,

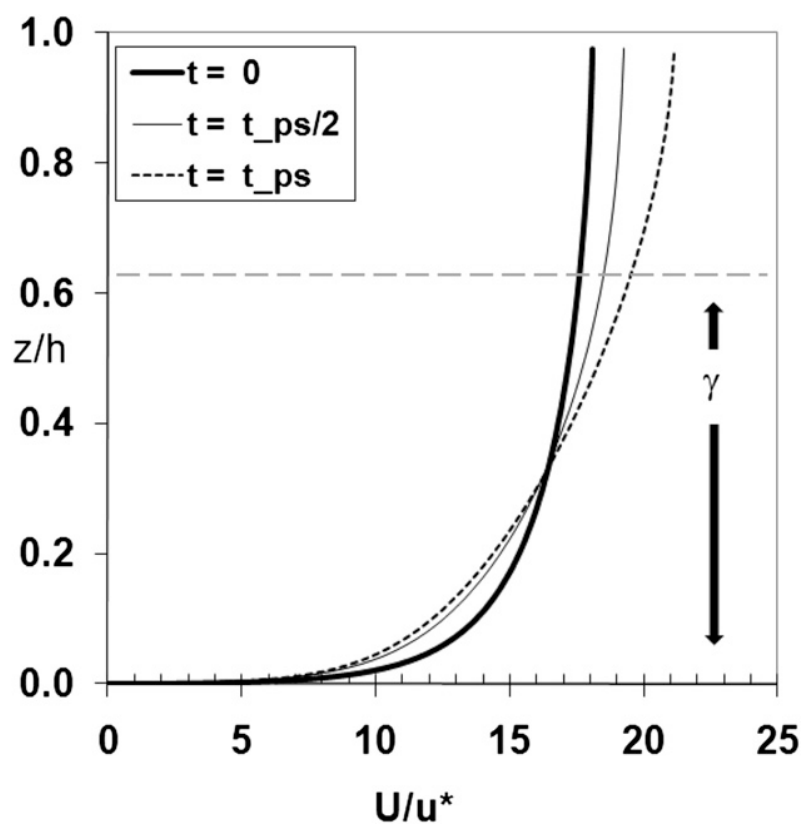

FIG. 8. Time evolution of the wind profiles for $h / L_{\mathrm{EXT}}=0.61$ during the interval $0<\hat{t}<\hat{t}_{\mathrm{PS}}$. At some level the profile intersects with the initial profile: this indicates the so-called velocity crossing point. Also the depth $\gamma$ of the PS layer is indicated (here $\sim 0.6$ ).

the profile at $t=t_{\mathrm{PS}} / 2$ crosses the initial profile. This socalled velocity crossing point (CP) appears to be constant in both height and time as it remains present at $t=t_{\mathrm{PS}}$. In turn, a stationary $\mathrm{CP}$ can be interpreted as a fixed velocity boundary condition for the lower part of the domain! As explained in section 2, such a condition effectively limits the downward heat transport to a maximum. A larger surface heat extraction results in excessive cooling. This essential insight learns that our pressure-driven flow is primarily subject to the same instability mechanism as the Couette flow (that is, in the period $0 \leq \hat{t} \leq \hat{t}_{\mathrm{PS}}$, of course).

It is evident that the $\mathrm{CP}$ and the momentum constraint in Eq. (19) are two sides of the same coin: the increasing static stability causes a steepening of the velocity profile. Because of momentum conservation, acceleration in the upper part of the PS layer must coincide with a deceleration at lower altitudes. Although "the conservation of momentum area" in principle could be realized with varying $\mathrm{CP}$, it is clear that the "freedom" of such $\mathrm{CP}$ to shift along the vertical is very limited. A linear velocity profile would, for example, imply no freedom at all.

In hindsight, the mechanism is summarized as follows: as a consequence of the difference in acceleration and diffusion time scales, a velocity crossing point emerges in instantaneously cooled pressure-driven flows. This CP acts as a fixed velocity boundary condition, which in turn 

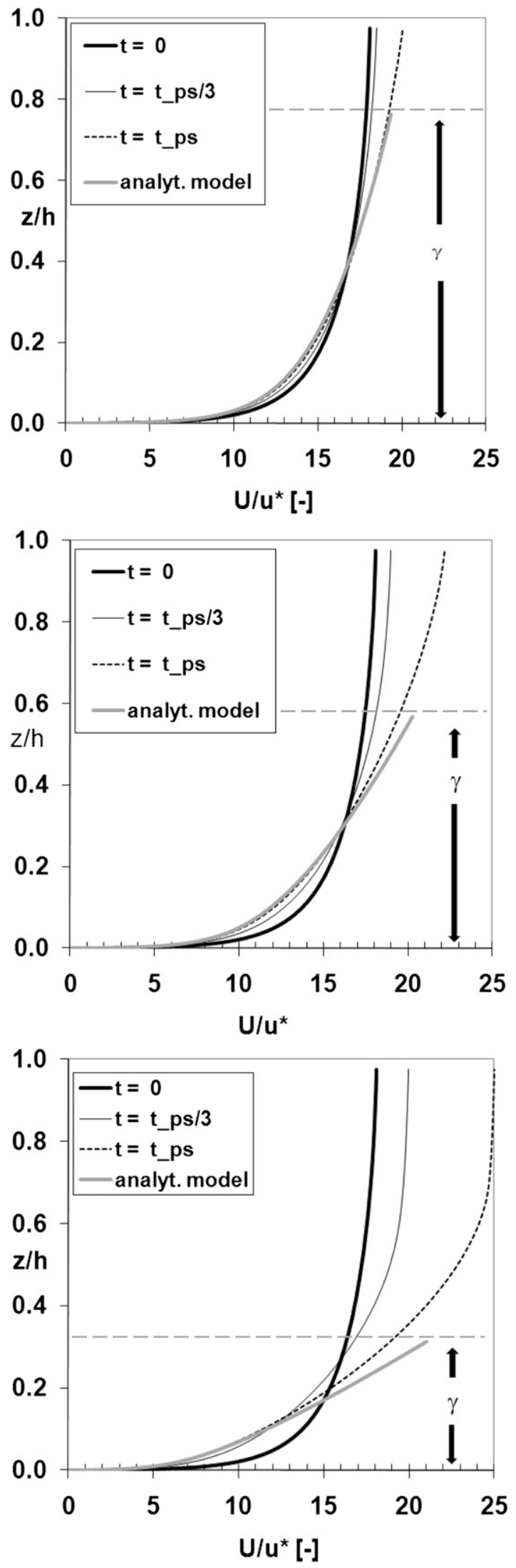

sets a limit on the maximum heat that can be transported toward the surface.

\section{Model validation}

\section{a. Pseudosteady profiles}

The evolution of the velocity profiles toward PSS is given in Figs. 9a-c. At the moment of minimum stress, $\hat{t}=\hat{t}_{\mathrm{PS}}$, analytical predictions (full gray) are compared to numerical solutions (dashed) for three cooling rates: $h / L_{\mathrm{EXT}}=0.31,0.61$, and 1.01 . As before, the analytical solution has validity for $z / h<\gamma$ only. For the lowest cooling rate [see Eq. (9a)], the agreement is most satisfying (as anticipated in appendix B). However, also for intermediate and higher cooling rates, the predictions seems reasonably close to the numerical solutions, particularly below the CP. For the latter cases, the agreement must be viewed in a relative sense: this means that one has to compare the differences between the prediction and the simulation below $z / h=\gamma$ with the momentum increasing over the time span between the initial and long-term steady states, which becomes increasingly larger with stability (appendix B). Nevertheless, the total momentum over the depth $z / h<\gamma$ seems to decrease a little in the period $\hat{t} \leq \hat{t}_{\mathrm{PS}}$. Therefore, assumption (19) must be viewed as a first-order approximation that facilitated understanding of the basic instability mechanism, rather than as an exact answer. For the examples the predicted height of the PS layer $\gamma$ and hence the predicted surface stress $\gamma=x^{2}=\left(u * 0 \mathrm{PS} / u *_{\mathrm{EXT}}\right)^{2}$ showed to be within $10 \%$ of the simulated values.

\section{b. The maximum sustainable heat flux in a channel flow}

By substituting Eqs. (17) and (18) in Eq. (19), one equation remains. The PSS model now relates the normalized friction velocity $x=u *_{0 \mathrm{PS}} / u_{* \mathrm{EXT}}$ to $h / L_{\mathrm{EXT}}$ in a similar way as expression (6) in section 2. Figure 10 depicts the solution curve for the neutral initial state. The qualitative similarity between this figure and Fig. 3 of section 2 is remarkable. Of course, both curves cannot be compared directly, as Fig. 10 uses $u_{*_{N}}$ instead of $u * \mathrm{EXT} ; h / L_{N}$ is generally much lower than $h / L_{\mathrm{EXT}}$,

FIG. 9. Development of the velocity profile simulated by the numerical model for the interval $0<\hat{t}<\hat{t}_{\mathrm{PS}}$. In solid gray the velocity profile by the analytical model is given. The depth of the PS layer is indicated by $\gamma$. Three stability classes are considered (top) $h / L_{\mathrm{EXT}}=0.31$, (middle) $h / L_{\mathrm{EXT}}=0.61$, and (bottom) $h / L_{\mathrm{EXT}}=1.01$. 


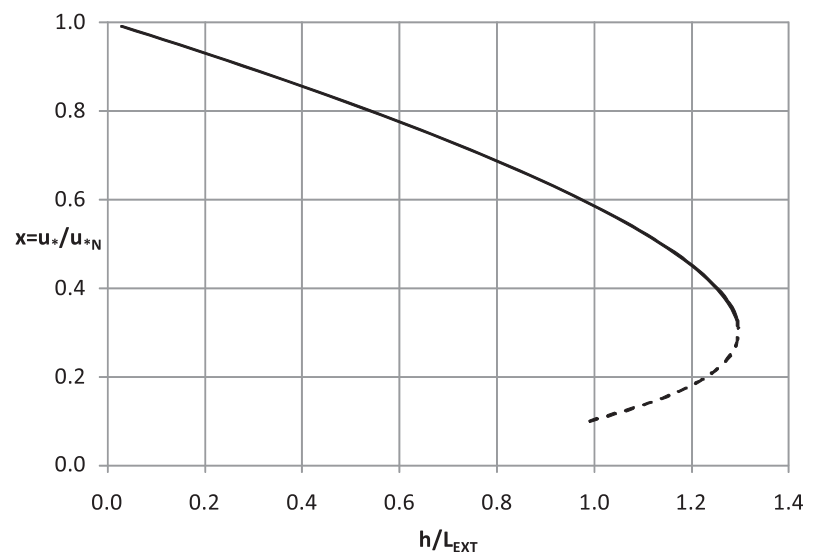

FIG. 10. "Parabolic" curve showing the extent of PSS space under neutral initial conditions, in terms of the surface friction velocity $x=u * 0 \mathrm{PS} / u * \mathrm{EXT}$ as a function of normalized cooling $h / L_{\text {EXT. }}$ The maximum heat flux that can be sustained by the flow for those initial conditions is predicted to be at $h / L_{\mathrm{EXT}}=1.29$. Note the qualitative similarity to Fig. 3, which was made for Couette flows. Again, the dashed part of the curve is considered to represent the part of the solution that is unstable to perturbations (see section 2).

because $u *_{N}$ is an upper estimate of actual friction velocity. For neutral initial conditions, the predicted maximum sustainable heat flux lies at $h / L_{\mathrm{EXT}}=1.29$. In view of the assumptions made, the critical value $h / L_{\mathrm{EXT}} \approx 1.29$ is acceptably close to the threshold found in the simulations, which indicated $h / L_{\mathrm{EXT}}=1.14$ (Fig. 5). We therefore conclude that the MSHF theory provides a useful framework in order to predict the collapse of turbulence in pressure-driven flows as well.

For completeness we note that the DNS simulations by Nieuwstadt (2005) and Flores and Riley (2011) indicated a lower critical value, $h / L_{\mathrm{EXT}}=0.4 \times 1.23 \approx 0.5$. The " 0.4 " correction is needed because Nieuwstadt's definition of $h / L$ did not include the von Kármán constant. The discrepancy between our results and the DNS can partly be explained by the viscous effects in the latter. Particularly for low Reynolds numbers, viscous dissipation of turbulence may enforce the collapse in response to initial suppression by stratification. Also, the actual value of $\alpha$ calculated from DNS seems to be somewhat higher than 5, particularly at weak stability, that is, below the point where collapse sets in $0<\mathrm{Ri}<1 / 3 \times(1 / \alpha)$ [section 2 ; compare also the large eddy simulation results by, e.g., Beare and Macvean (2004)]. For higher $\alpha$, the predicted $\left(h / L_{\mathrm{EXT}}\right)$-critical decreases: $h / L_{\text {EXT,Crit }} \propto 1 / \alpha$. In any case, heat transport limitations are expected to play a qualitatively similar role in stratified DNS studies, particularly because a recent comparison between the local similarity model and a DNS by the current authors (Moene et al. 2010) revealed a close correspondence with respect to the

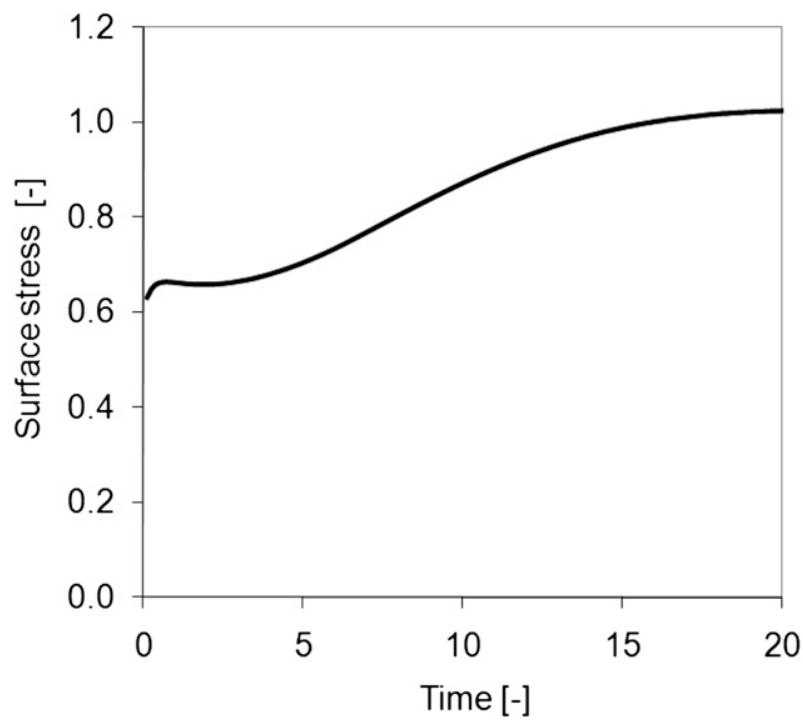

FIG. 11. Normalized surface stress vs time. A PSS for $h / L_{\mathrm{EXT}}=0.61$ is taken as the initial condition.

mean and the flux profiles for the present channel flow configuration. Additional simulations with a high Reynolds number DNS may help to clarify this point in the future.

\section{c. Pseudosteadiness as a preferred state}

As collapse is predicted in terms of cooling and initial momentum, the model predicts that nonneutral initial velocity profiles will lead to different critical $h / L_{\text {EXT. }}$. For brevity reason this interesting aspect will not be discussed here. We will, however, apply the pseudosteady state as an alternative initial condition. The reason for this is to check whether the pseudosteady state is really a preferred intermediate state. Of course, by definition it has the same momentum as the neutral initial case (and hence the same $h / L_{\text {EXT }}$-critical). Now, if such a thing as pseudosteadyness really exists, the system should have a preference to remain in such state for a while. Figure 11 shows the normalized surface friction velocity as a function of time with the PS initial state for $h / L_{\mathrm{EXT}}=$ 0.61. Apart from a small initial disturbance (PSS is an approximation), there is a clear tendency for the flux to remain constant. For larger times the system is attracted to the long-term steady-state solution, so that finally a typical S curve appears with two flat, "stationary" levels.

The tendency of the system to be in balance with this PS initial condition is even more evident from the velocity profiles depicted in Fig. 12. For short times the system in the lower domain has negligible tendency, as it is "comfortable" over the depth of the PS layer $(z / h \leq 0.6)$. During this period, significant acceleration 


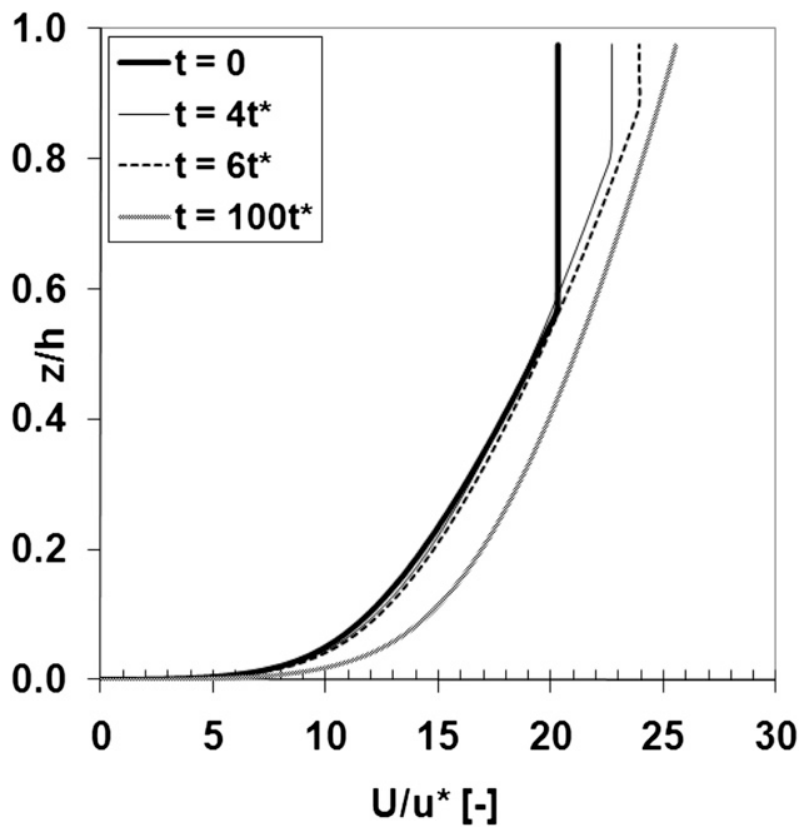

FIG. 12. Initial stage of velocity profile dynamics. Starting from pseudosteady equilibrium $t / t_{*}=0$ toward its long-term equilibrium $t / t_{*}=100$

only occurs in the upper, decoupled domain (appendix B). At later stage, sufficient time for acceleration has elapsed and the total domain is adjusted in equilibrium with the forcings.

\section{Atmospheric implications}

\section{a. The velocity crossing point}

The flow configuration used in the present study is a simplification of complex reality. Nevertheless, it is expected that the essential collapse mechanisms will also play a role in the atmosphere. For example, it can be anticipated that the typical diffusion time for nocturnal boundary layers is much shorter than the acceleration time. The magnitude of those time scales can be estimated from typical values. If we take $200 \mathrm{~m}$ as a typical $h$ and a value of $0.25 \mathrm{~m} \mathrm{~s}^{-1}$ for the friction velocity and use $\tau_{\text {diff }} \cong h^{2} /\left(u_{*} h\right)=h / u_{*}$, we obtain a diffusion time scale on the order of 10-15 min. This is relatively small compared to the typical acceleration time scale $f^{-1}$, which is on the order of hours at midlatitudes (the Coriolis parameter $f$ has a typical magnitude of $10^{-4} \mathrm{~s}^{-1}$ ).

The separation of time scales implies that the lower, coupled part of the nocturnal boundary layer has to "survive' on the available momentum present at the onset of cooling. This initial momentum is largely determined by the magnitude of the geostrophic wind (Fig. 1). As before, momentum conservation facilitates the

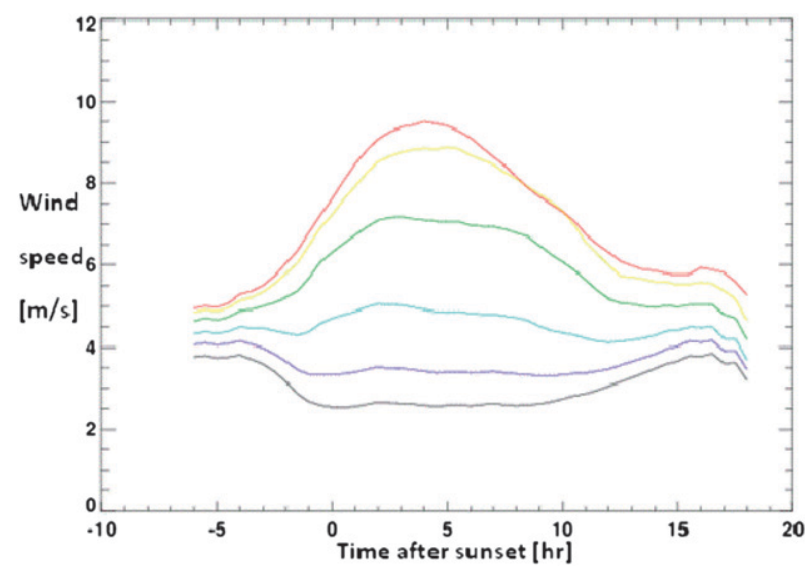

FIG. 13. Composite average of the diurnal cycle of the wind speed, under clear-sky situations at Cabauw with geostrophic winds between 5 and $10 \mathrm{~m} \mathrm{~s}^{-1}$ (see text). Line colors defined as red: $200 \mathrm{~m}$, yellow: $140 \mathrm{~m}$, green: $80 \mathrm{~m}$, light blue: $40 \mathrm{~m}$, dark blue: $20 \mathrm{~m}$, and black: $10 \mathrm{~m}$.

existence of a level with constant velocity. Indeed, also in the atmosphere, existence of such a velocity crossing point can be anticipated: high-level nocturnal winds (say, $>100 \mathrm{~m}$ ) are often subject to accelerations in response to decoupling, which leads to inertial oscillations (Blackadar 1957; Shapiro and Fedorovich 2010). In contrast, near-surface winds (say, $<20 \mathrm{~m}$ ) usually become weaker during the evening. Consequently, at intermediate levels $(\sim 20-50 \mathrm{~m})$ the wind tends to stay constant in magnitude after sunset (Wieringa 1989). Note that, although changes in midlevel magnitudes are expected to be small, rotational effects due to inertial oscillation may still play a role at all levels (Baas et al. 2012).

A composite average of the diurnal wind cycle at Cabauw for different levels is given in Fig. 13. For 28 clear nights with geostrophic winds between 5 and $10 \mathrm{~m} \mathrm{~s}^{-1}$, wind speeds were averaged at each observed level (as in Baas et al. 2012). The upper levels, 140 and $200 \mathrm{~m}$, become largely decoupled from the surface and show clear inertial oscillations. This decoupling results from the fact that cold air is diffused upward rapidly, whereas reestablishment of shear is much slower. At the lower levels, 10 and $20 \mathrm{~m}$, a decrease of the wind magnitude is noticeable. The combined effect results in a visual "butterfly wing" (Fig. 13), which is typical for diurnal-wind observation over homogeneous, flat terrain [a clear example for the Wangara experiment is given in Mahrt (1981)]. Finally, the 40-m wind takes the intermediate path and stays relatively constant in magnitude (near $50 \mathrm{~m}$ for Wangara). As wind shear over the 40-m bulk layer is approximately fixed, the sustainable heat flux in the nocturnal boundary layer is limited to 
a well-defined maximum at given geostrophic wind. In the companion paper (Van de Wiel et al. 2012), this aspect is elaborated in order to predict the occurrence of very stable nights observed at Cabauw.

\section{b. The critical geostrophic wind speed}

However, also at this stage it is tempting to reflect on Fig. 1 given in the introduction. To this end let us naively use Eq. (3) as a predictive tool for the maximum sustainable heat flux. This expression is based on Monin-Obukhov similarity scaling, so that it does not incorporate the effects of vertical flux divergence or wind turning. Next, we use the fact that the wind at the crossing point cannot exceed the ambient geostrophic wind, thus $\Delta U<G$. Assuming parameter values $\left(\rho, c_{p}, \kappa, \alpha, \theta_{\text {ref }}, g, z_{0}, h \equiv z_{\text {cross }}\right)=$ $(1.2,1005,0.4,5,285,9.81,0.01,40)$, we evaluate the lowest three wind categories of Fig. $1, G=(3,6,9) \mathrm{m} \mathrm{s}^{-1}$, and find the upper estimates for the maximum sustainable heat flux: $\left|H_{\mathrm{MAX}}\right|<(2,13,44) \mathrm{W} \mathrm{m}^{-1}$. Even with this crude estimate, it is clear that a geostrophic speed of $3 \mathrm{~m} \mathrm{~s}^{-1}$ is unlikely to be sufficient to sustain continuous turbulence. For $G=9 \mathrm{~m} \mathrm{~s}^{-1}, H_{\mathrm{MAX}}$ amounts to $44 \mathrm{~W} \mathrm{~m}^{-2}$, which is of the same order of magnitude as the net radiation itself, so that sustained turbulence is likely to be realizable. A further inspection of intermediate $G$ values leads to the typical range $4<G<$ $8 \mathrm{~m} \mathrm{~s}^{-1}$ for the expected transition between the VSBL and the VSBL over homogeneous grassland. Note that this range is in agreement with the range for decoupling found in a recent study by Acevedo et al. (2012). Their study indicates a transition for $5<G<7 \mathrm{~m} \mathrm{~s}^{-1}$, irrespective of specific model details. Though their results are not directly transferable to the present study, for example, because temperature boundary conditions are used at the surface (see below), the robustness of their results can be anticipated from our analysis: $H_{\text {MAX }}$ depends in a cubic way on the forcing wind speed [Eq. (3)]. This high sensitivity implies that the wind speed range for which $H_{\mathrm{MAX}}$ goes from negligible to significant values is very narrow [see the companion paper (Van de Wiel et al. 2012) for more details].

\section{c. Boundary layer decoupling}

Because of the simplified character of the model system in the present study, a direct quantitative comparison with atmospheric data is not possible. However, some of the qualitative findings appear to be supported by some observational studies by others. For example, according to the present study, a two-layer system appears to evolve at larger cooling rates: an upper layer that becomes (temporarily) decoupled and is governed by acceleration processes, and a lower layer that stays in contact with the ground by turbulent diffusion (Figs. 7, 9c). This two-layer structure resembles the schematic picture for very stable boundary layers that was proposed by Banta et al. (2007). Based on a thorough investigation on Cooperative Atmosphere-Surface Exchange Study1999 (CASES-99) nights, they subdivided the lower atmosphere into a shallow attached boundary layer (BL), a quiescent layer $(\mathrm{Q})$, and a deeper, overlying part (A) that may include remnants of the afternoon mixed layer (not considered here). Interestingly, observations over complex topography have shown that both coupling and decoupling may even occur simultaneously at different locations (Acevedo and Fitzjarrald 2003). In any case, the decoupled state favors an increasing bulk shear that may erode the decoupled state itself by regeneration of some turbulence in the long term (Businger 1973). A very instructive example of this aspect was given by Schubert (1977), who used acoustic soundings. For several nights, the initial quasi-laminar state transformed to a turbulent end state of the BL in response to gradual flow acceleration [as in Fig. 5; see also McNider and Pielke (1981)]. Finally, an interesting spatial analogy to temporary decoupling was given by Smedman et al. (1997). They studied warm-air advection over cold sea and showed how an initially strongly stratified internal boundary layer evolved toward a well-mixed layer at larger distances.

\section{Conclusions}

The collapse of turbulence in the period after the onset of cooling can be understood from the fact that the time scale for boundary layer diffusion is much smaller than the time scale for flow acceleration. Available momentum in the layer adjacent to the surface is rapidly redistributed by diffusion in order to achieve a temporary force balance in this layer. This state is referred to as the pseudosteady state. At higher levels, insufficient shear is available to maintain turbulence. This region becomes temporary decoupled from the flow below and slowly accelerates.

Analytical expressions for the pseudosteady state were found by extending Monin-Obukhov similarity profiles to the general case that includes vertical flux divergence. In the absence of significant acceleration, the pseudosteady layer has to "survive" on initial available momentum. This kinetic energy constraint implies that the heat that can be transported to the surface is also limited to a certain maximum in this period. In case the surface heat extraction exceeds this limit, the inversion rapidly increases so that turbulence is bound to collapse due to the intense density stratification. The mechanism is fully analogous to the one described in VDW07 for Couette flows. Here, the theory is supported by numerical 
simulations of a cooled channel flow. Simulated velocity profiles and critical cooling rates are predicted in close agreement with the simulations.

With respect to atmospheric implications, the initial momentum of the flow will be determined by the geostrophic wind. In turn, the energy demand at the surface is largely influenced by the nocturnal longwave radiative forcing. From the present study, the emergence of the very stable boundary layer as a distinct, separate flow regime can now be explained by the interplay between those external factors. In the companion paper (Van de Wiel et al. 2012), the insights are used to formulate a simple, practical model that can be used to predict the occurrence of both weakly and very stable nights. From this, the authors strongly encourage future studies on regime transition with large-eddy simulations that explore sensitivity to geostrophic wind forcing in the range 3-7 m s ${ }^{-1}$, similar to Jiménez and Cuxart (2005), possibly with improved subgrid closures as in Zhou and Chow (2011). In order to be representative of the present model, the simulations should be flux driven from below. This means that a prescribed surface flux should be implied [unlike in the intercomparison of the first Global Energy and Water Cycle Experiment Atmospheric Boundary Layer Study (GABLS1) (Beare et al. 2006; Acevedo et al. 2012), or that a simple surface energy balance should be included (Basu et al 2008)]. As discussed in the companion paper (Van de Wiel et al. 2012), a strongly isolating surface with low heat capacity, such as fresh snow, is preferred for the latter case, as to eliminate the dominance of soil heat transport or heat storage.

Acknowledgments. We are thankful to the Royal Netherlands Meteorological Institute (KNMI), in particular to Dr. Fred Bosveld and Dr. Peter Baas, for having provided the observational data presented in illustrations 1 and 13. The useful comments on earlier versions of the manuscript by Peggy Lemone, Larry Mahrt, Bert Holtslag, Sukanta Basu, Peter Baas, and Jielun Sun are highly appreciated. The research has been supported by a VIDI Grant (10011425) from the Dutch National Science Foundation (NWO), which is gratefully acknowledged.

\section{APPENDIX A}

\section{Quasi-Steady Solutions of Homogenous, 1D Pressure-Driven, Stratified Flow}

The point of departure is Eqs. (14) and (15): $u_{*}^{2} / u_{* \mathrm{EXT}}^{2}=(1-z / h), H / H_{0}=(1-z / h)$. We define the steady-state Obukhov length and its local equivalent as

$$
L_{\mathrm{EXT}}=\frac{u_{* \mathrm{EXT}}^{2}}{\theta_{* \mathrm{EXT}}} \frac{T_{\text {ref }}}{\kappa g} ; \quad \Lambda=\frac{u_{*}^{2}}{\theta_{*}} \frac{T_{\text {ref }}}{\kappa g}
$$

with $\theta *_{\mathrm{EXT}} \equiv H_{0} / \rho c_{p} u *_{\mathrm{EXT}}$. Through the flux profiles, both definitions are related as shown:

$$
\Lambda=L_{\mathrm{EXT}} \sqrt{1-z / h} .
$$

The conclusions given above are independent of turbulent closure. To arrive at the equilibrium profiles of $U$ and $T$, specific closure assumptions relating local fluxes to gradients have to be adopted. Here, we adopt loglinear similarity functions as shown:

$$
\frac{d U}{d z} \frac{\kappa z}{u_{*}}=1+\alpha \frac{z}{\Lambda} ; \quad \frac{d T}{d z} \frac{\kappa z}{\theta_{*}}=1+\alpha \frac{z}{\Lambda}
$$

After inserting Eq. (A1) and normalizing by $u *$ EXT, $\theta *_{\mathrm{EXT}}$, and $h$, defining $q \equiv z / h$, we find

$$
\frac{d \hat{u}}{d q}=\frac{\sqrt{1-q}}{\kappa q}+\frac{\alpha}{\kappa} \frac{h}{L_{\mathrm{EXT}}} ; \quad \frac{d \hat{\theta}}{d q}=\frac{\sqrt{1-q}}{\kappa q}+\frac{\alpha}{\kappa} \frac{h}{L_{\mathrm{EXT}}} .
$$

Integrating the wind profile gives

$$
\hat{u}=\frac{1}{\kappa}\left(2 \sqrt{1-q}-2 \operatorname{atanh} \sqrt{1-q}+\alpha q \frac{h}{L_{\mathrm{EXT}}}\right)-C_{1} .
$$

The constant is readily found from $\hat{u}=0$ at $q=q_{0}$. For temperature, the integration constant results from the energy constraint: total cooling should balance the extracted heat; hence,

$$
\hat{\theta}=\frac{1}{\kappa}\left(2 \sqrt{1-q}-2 \operatorname{atanh} \sqrt{1-q}+\alpha q \frac{h}{L_{\mathrm{EXT}}}\right)-C_{2}
$$

with the energy constraint

$$
\int_{q_{0}}^{1} \hat{\theta} d q=-t / t_{*}, \quad \text { with } \quad t_{*}=\sqrt{h /\left(-\frac{1}{\rho} \frac{\partial P}{\partial x}\right)},
$$

so that $C_{2}$ is found as shown:

$$
\begin{aligned}
C_{2}= & \frac{t / t_{*}+A}{1-q_{0}} \text { with } \\
A= & \frac{1}{\kappa}\left[\frac{4}{3}\left(1-q_{0}\right)^{3 / 2}-2\left(1-q_{0}\right)^{1 / 2}\right. \\
& \left.+\frac{\alpha}{2} \frac{h}{L_{\mathrm{EXT}}}\left(1-q_{0}^{2}\right)+q_{0} \ln \left(\frac{1+\sqrt{1-q_{0}}}{1-\sqrt{1-q_{0}}}\right)\right] .
\end{aligned}
$$


The analytical solutions where validated using longterm $\left(t / t_{*}=100\right)$ numerical integrations.

\section{Monin-Obukhov similarity as an asymptotic case of local scaling}

Interestingly, it can be shown that the general result, Eqs. (A4) and (A5), degenerate asymptotically to $\mathrm{MO}$ similarity in the limit $q \downarrow q_{0}$ with $q_{0} \ll 1$, that is, when approaching the surface. We use the fact that $\operatorname{atanh}(y)=(1 / 2) \ln [(1+y) /(1-y)]$ for $y<1$. This gives

$$
\hat{u}=\frac{1}{\kappa}\left[2 \sqrt{1-q}-\ln \left(\frac{1+\sqrt{1-q}}{1-\sqrt{1-q}}\right)+\alpha q \frac{h}{L_{\mathrm{EXT}}}\right]_{q_{0}}^{q}
$$

for $q \ll 1$; that is, with $\sqrt{1-q} \approx 1-(1 / 2) q$ we retrieve $\hat{u} \approx(1 / \kappa)\left[\ln \left(q / q_{0}\right)+\alpha q h / L_{\mathrm{EXT}}\right]$, which is the traditional log-linear MO profile! Note that it is a natural asymptotic result of local similarity, whereas the existence of MO similarity is often misunderstood from the existence of a true "constant flux" layer. In reality, flux divergences cannot be zero in steady pressuredriven flows. Stress divergence is needed to oppose a finite pressure gradient [Eq. (8)]. In turn, heat flux divergence is needed for boundary layer cooling [Eq. (9)]. However, close to the surface the relative change of flux with height is still small, which explains the success of MO scaling as an approximation [apart from the effects of compensating errors discussed in Delage (1997)].

\section{APPENDIX B}

\section{Time-Scale Analysis and Consequences}

Here, it is shown how a momentum constraint appears from the fact that the typical diffusion timescale $\tau_{\text {diff }}$ for building up the PS layer in accordance with the new surface boundary condition is much smaller than the typical acceleration time scale $\tau_{\text {acc }}$ to reach long-term steady state. We start with a lower-bound estimate for $\tau_{\text {acc }}$. The typical velocity increment (averaged over the channel depth) from a neutral state to a stably stratified steady state is estimated by using Monin-Obukhov theory (for simplicity MO is preferred over local scaling) as shown:

$$
\begin{aligned}
U(t=0) & =\left(u_{* \mathrm{EXT}} / \kappa\right)\left[\ln \left(z / z_{0}\right)\right] \\
U(t \rightarrow \infty) & =\left(u_{*{ }_{\mathrm{EXT}}} / \kappa\right)\left[\ln \left(z / z_{0}\right)+\alpha z / L_{\mathrm{EXT}}\right],
\end{aligned}
$$

so that

$$
\langle\Delta U\rangle=\frac{\alpha u_{* \mathrm{EXT}} h}{2 \kappa L_{\mathrm{EXT}}},
$$

with $\langle\Delta U\rangle$ as the height-averaged velocity difference. The characteristic acceleration time scale is estimated from the height-integrated momentum balance (per height) as shown:

$$
\frac{\langle\Delta U\rangle}{\tau_{\mathrm{acc}}}=-\frac{1}{\rho} \frac{\partial P}{\partial x}=\frac{u_{* \mathrm{EXT}}^{2}}{h}-\frac{u_{*_{0}}^{2}(t)}{h} .
$$

By replacing the time-dependent surface friction by its minimum value, namely $\gamma u_{* \mathrm{EXT}}^{2}$, a lower (i.e., conservative) bound for acceleration time scale is found as shown:

$$
\tau_{\mathrm{acc}}=\frac{\alpha}{2 \kappa} \frac{h^{2}}{u_{* \mathrm{EXT}} L_{\mathrm{EXT}}} \frac{1}{(1-\gamma)} .
$$

In the meantime, a pseudosteady layer tries to develop from below. As argued below, this layer is decoupled from the upper boundary layer. By rapid redistribution (diffusion) of the initial momentum, it seeks a balance with the new surface boundary condition and the reduced depth of the turbulent layer. As a result the surface friction velocity is reduced to $u_{*}=\sqrt{-(1 / \rho)(\partial P / \partial x) \gamma h}$ compared to its original $u_{*}=u_{* \mathrm{EXT}}=\sqrt{-(1 / \rho)(\partial P / \partial x) h}$. The time scale for this process is estimated by dividing the square of the characteristic length scale by the eddy diffusivity as follows:

$$
\tau_{\text {diff }}=\frac{\gamma^{2} h^{2}}{\gamma^{2} \kappa u_{* \mathrm{EXT}} L_{\mathrm{EXT}}}=\frac{h^{2}}{\kappa u_{* \mathrm{EXT}} L_{\mathrm{EXT}}} .
$$

Finally, both time scales are compared as shown:

$$
\frac{\tau_{\text {diff }}}{\tau_{\text {acc }}}=\frac{2}{\alpha}(1-\gamma)=0.4 \times(1-\gamma)
$$

Again, this is an upper bound by the conservative estimate of surface friction in $\tau_{\text {acc. }}$. As such, it appears that the system is able to reach a PSS in the lower domain before the overall steady state sets in. As will be shown, in this region there is sufficient initial momentum to distribute in order to reach a PSS. At the higher levels, there is certainly insufficient momentum in the initial flow, so that rapid redistribution by diffusion cannot help (Fig. 6). This part of the flow has to wait until 


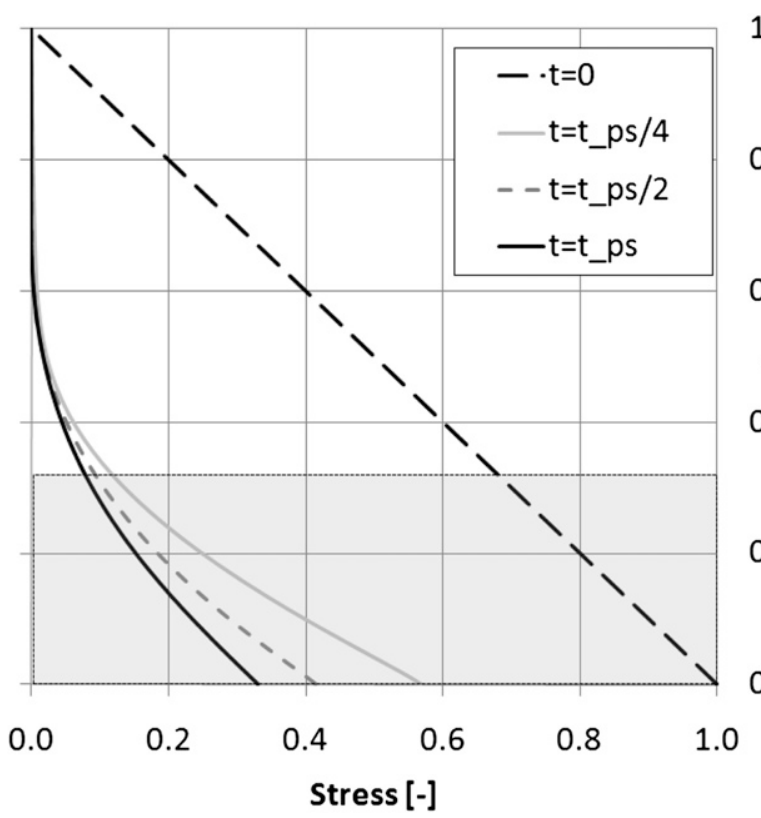

FIG. B1. Development of the normalized stress profile $u_{*_{0}}^{2} / u_{* \text { EXT }}^{2}$ in the period $0<\hat{t}<\hat{t}_{\mathrm{PS}}$. The depth $\gamma$ of the PS layer is indicated by the gray box.

sufficient time of acceleration has passed. Although there is no access of momentum that can be diffused upward, there is a new pool of cold air to diffuse. Therefore, at higher levels the temperature gradients also increase, but the shear is still close to its neutral initial value. As the increase of temperature gradient scales with $\tau_{\text {diff }}$ and the increase of shear scales with $\tau_{\text {acc }}$, the high-level Richardson numbers will be large for $t \ll \tau_{\text {acc }}$ and the upper layer temporarily decouples from the lower layer. Consequently, at the top of the domain a near-frictionless acceleration occurs, as illustrated in Figs. 8, 9, and 12. The separation of time scales, $\tau_{\text {diff }} \ll \tau_{\text {acc }}$, leads to an important momentum constraint of the flow, which ultimately limits the downward heat flux to a maximum (main text). In line with the discussion above, we take $t_{\mathrm{PS}} \cong \tau_{\text {diff }}$ and depart from the momentum equation as shown:

$$
\frac{\partial U}{\partial t}=\frac{u_{* \operatorname{EXT}}^{2}}{h}+\frac{\partial u_{*}^{2}}{\partial z}
$$

Integration with respect to the depth of the pseudosteady layer $\gamma h$ and time until PSS $t_{\mathrm{PS}}$ gives

$$
\begin{aligned}
& \int_{z_{0}}^{\gamma h}\left[U(t=0)-U\left(t=t_{\mathrm{PS}}\right)\right] d z \\
& \quad=\gamma u_{* \mathrm{EXT}}^{2} t_{\mathrm{PS}}+\int_{0}^{t_{\mathrm{PS}}}\left[u_{*}^{2}(z=\gamma h)-u_{* 0}^{2}\right] d t .
\end{aligned}
$$

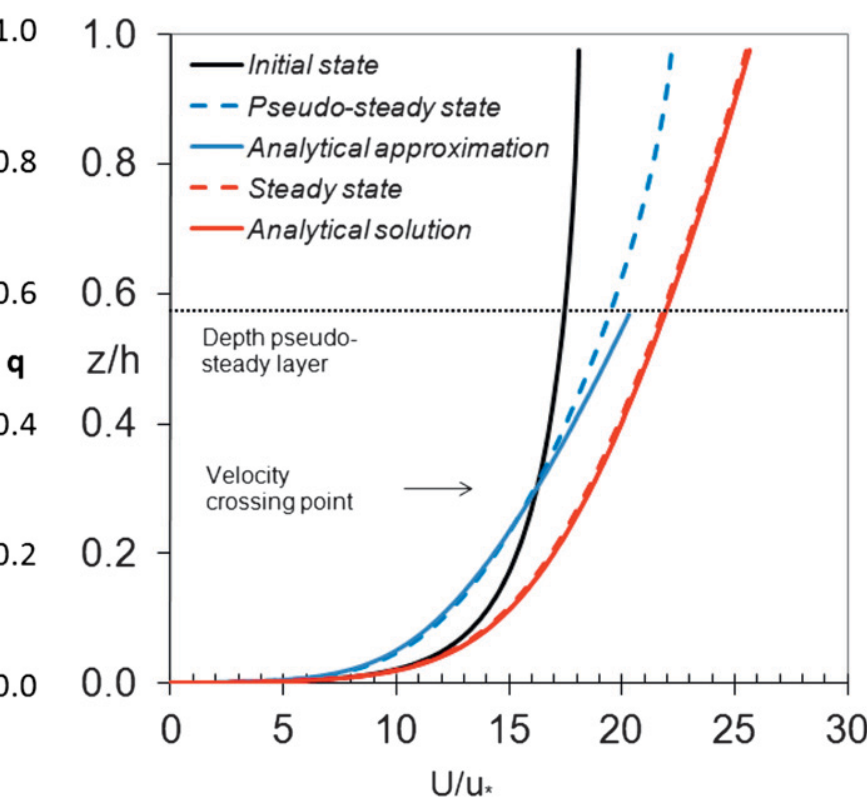

FIG. B2. Illustration $(h / L=0.61)$ of the fact that the relative deceleration during $0<\hat{t}<\hat{t}_{\mathrm{PS}}$ for $z / h<\gamma$ is small: the heightintegrated difference over $0<z / h<\gamma$ between the true profile at PSS from the numerical simulations (blue dashed) and the prediction that assumes the exact conservation of initial momentum (solid blue) is small compared to the total acceleration over this layer that occurs between initial state (black) and final state (red).

The last term describes the effect of friction over the PS layer. Figure B1 shows the evolution of normalize turbulent stress in case $h / L_{\mathrm{EXT}}=1.01$. Interestingly, the stress profile rapidly evolves to a slope close to $45^{\circ}$ (it has relatively little "freedom" as both the initial and PS states have this slope). In the limit case of a timeindependent slope of $45^{\circ}$, the integral at the rhs becomes $-\gamma u_{* \mathrm{EXT}}^{2} t_{\mathrm{PS}}$, so that the total rhs becomes zero. This would directly imply that the height-integrated momentum over the PS layer remains constant during this phase.

However, in the initial phase, $t<t_{\mathrm{PS}} / 4$, the slope seems to be steeper than $45^{\circ}$, in a sense that some net deceleration occurs over this period. We compare the maximum possible deceleration in the PS layer to the total acceleration that will occur over this part of the domain. It shows that even in this worst-case scenario, deceleration effects are relatively small, so that the conservation of initial momentum is supported again.

Inspection of Fig. B1 learns that the upper bound of the frictional term in Eq. (B7) is $-t_{\mathrm{PS}} u_{* \mathrm{EXT}}^{2}$, that is, as if the turbulence were not reduced and fully diverges over this small depth $\gamma h$. We normalize this maximum error by dividing Eq. (B7) with respect to $\gamma h\langle\Delta U\rangle$, the total long-term momentum increase over depth (Fig. B2) as shown: 


$$
\frac{\int_{z_{0}}^{\gamma h}\left[U(t=0)-U\left(t=t_{\mathrm{PS}}\right)\right] d z}{\gamma h\langle\Delta U\rangle}=-\frac{(1-\gamma) u_{*}^{2} \mathrm{EXT}_{\mathrm{PS}}}{\gamma h\langle\Delta U\rangle} .
$$

Next, we realize that $t_{\mathrm{PS}} \cong \tau_{\text {diff }}$ and we insert Eqs. (B1) and (B4), as follows:

$$
\frac{\int_{z_{0}}^{\gamma h}\left[U(t=0)-U\left(t=t_{\mathrm{PS}}\right)\right] d z}{\gamma h\langle\Delta U\rangle}=-\frac{2}{\alpha} \frac{(1-\gamma)}{\gamma}=-\frac{1}{\gamma} \frac{\tau_{\mathrm{diff}}}{\tau_{\mathrm{acc}}} .
$$

This important result reveals that the relative change in momentum (deceleration) is expected to be small because $\tau_{\text {diff }} \ll \tau_{\text {acc }}$. This relatively small change can be explained by the fact that frictional term and the pressure gradient term in Eq. (B7) largely cancel out. The relative deceleration will increase for decreasing $\gamma$, as confirmed by Figs. 9a-c in the main text. Note that the prefactor $1 / \gamma$ is still bounded by the smallest $\gamma[O(0.1-$ $0.2)]$, which allows for continuous turbulence, that is, without collapse. It is concluded that the conservation of momentum during $0 \leq t \leq t_{\mathrm{PS}}$ follows from the fact that $\tau_{\text {diff }} \ll \tau_{\text {acc }}$, and the pseudosteady state solution is to a good approximation constraint by

$$
\int_{z_{0}}^{\gamma h}\left[U(t=0)-U\left(t=t_{\mathrm{PS}}\right)\right] d z \approx 0 .
$$

This result is used in the main text.

\section{REFERENCES}

Acevedo, O. C., and D. R. Fitzjarrald, 2003: In the core of the night - Effects of intermittent mixing on a horizontally heterogeneous surface. Bound.-Layer Meteor., 106, 1-33.

— , F. D. Costa, and G. A. Degrazia, 2012: The coupling state of an idealized stable boundary layer. Bound.-Layer Meteor., 145, 211-228, doi:10.1007/s10546-011-9676-3.

Baas, P., B. J. H. van de Wiel, L. van den Brink, and A. A. M. Holtslag, 2012: Composite hodographs and inertial oscillations in the nocturnal boundary layer. Quart. J. Roy. Meteor. Soc., 138, 528-535, doi:10.1002/qj.941.

Baklanov, A. A., and Coauthors, 2011: The nature, theory, and modeling of atmospheric planetary boundary layers. Bull. Amer. Meteor. Soc., 92, 123-128.

Banta, R. M., 2008: Stable-boundary-layer regimes from the perspective of the low-level jet. Acta Geophys., 56, 58-87.

—, L. Mahrt, D. Vickers, J. Sun, B. B. Balsley, Y. L. Pichugina, and E. J. Williams, 2007: The very stable boundary layer on nights with weak low-level jets. J. Atmos. Sci., 64, 3068-3090.

Basu, S., F. Porté-Agel, E. Foufoula-Georgiou, J.-F. Vinuesa, and M. Pahlow, 2006: Revisiting the local scaling hypothesis in stably stratified atmospheric boundary layer turbulence: An integration of field and laboratory measurements with large-eddy simulations. Bound.-Layer Meteor., 119, 473500.

A. A. M. Holtslag, B. J. H. van de Wiel, A. F. Moene, and G.-J. Steeneveld, 2008: An inconvenient "truth" about using sensible heat flux as a surface boundary condition in models under stably stratified regimes. Acta Geophys., 56, 88-99.

Beare, R. J., and M. K. Macvean, 2004: Resolution sensitivity and scaling of large-eddy simulations of the stable boundary layer. Bound.-Layer Meteor., 112, 257-281.

— simulations of the stable boundary layer. Bound.-Layer Meteor., 118, 247-272.

Beljaars, A. C. M., and A. A. M. Holtslag, 1991: Flux parameterization over land surfaces for atmospheric models. J. Appl. Meteor., 30, 327-341.

— , and P. Viterbo, 1998: Role of the boundary layer in a numerical weather prediction model. Clear and Cloudy Boundary Layers: Proceedings of the Colloquium Clear and Cloudy Boundary Layers, A. A. M. Holtslag, P. G. Duynkerke, and P. J. Jonker, Eds., Royal Netherlands Academy of Arts and Sciences, 287-304.

Blackadar, A. K., 1957: Boundary layer wind maxima and their significance for the growth of nocturnal inversions. Bull. Amer. Meteor. Soc., 38, 283-290.

Businger, J. A., 1973: Turbulent transfer in the atmospheric surface layer. Workshop on Micrometeorology, D. A. Haugeri, Ed., Amer. Meteor. Soc., 67-98.

Cuxart, J., and Coauthors, 2006: Single-column model intercomparison for a stably stratified atmospheric boundary layer. Bound.-Layer Meteor., 118, 427-432.

De Bruin, H. A. R., 1994: Analytic solutions of the equations governing the temperature fluctuation method. Bound.-Layer Meteor., 68, 427-432.

Delage, Y., 1997: Parameterising sub-grid scale vertical transport in atmospheric models under statistically stable conditions. Bound.-Layer Meteor., 82, 23-48.

—, P. A. Bartlett, and J. H. McCaughey, 2002: Study of 'soft' night-time surface-layer decoupling over forest canopies in a land-surface model. Bound.-Layer Meteor., 103, 253276.

Derbyshire, S. H., 1990: Nieuwstadt's stable boundary layer revisited. Quart. J. Roy. Meteor. Soc., 116, 127-158.

_ , 1999a: Boundary-layer decoupling over cold surfaces as a physical boundary-instability. Bound.-Layer Meteor., 90, 297-325.

__ 1999b: Stable boundary layer modeling: Established approaches and beyond. Bound.-Layer Meteor., 90, 423446.

Edwards, J. M., 2009: Radiative processes in the stable boundary layer: Part II. The development of the nocturnal boundary layer. Bound.-Layer Meteor., 131, 127-146.

England, D. E., and R. T. McNider, 1995: Stability functions based upon shear functions. Bound.-Layer Meteor., 74, 113130.

Estournel, C., and D. Guedalia, 1985: Influence of geostrophic wind on atmospheric nocturnal cooling. J. Atmos. Sci., 42, 2695-2700.

Fernando, H. J. S., and J. C. Weil, 2010: Whither the stable boundary layer? Bull. Amer. Meteor. Soc., 91, 1475-1484.

Flores, O., and J. J. Riley, 2011: Analysis of turbulence collapse in the stably stratified surface layer using direct numerical simulation. Bound.-Layer Meteor., 139, 241-259. 
Galperin, B., S. Sukoriansky, and P. S. Anderson, 2007: On the critical Richardson number in stably stratified turbulence. Atmos. Sci. Lett., 8, 65-69.

Grachev, A. A., C. W. Fairall, P. O. G. Persson, E. L. Andreas, and P. S. Guest, 2005: Stable boundary-layer regimes: The SHEBA data. Bound.-Layer Meteor., 116, 201-235.

—, E. L. Andreas, C. W. Fairall, P. S. Guest, and P. O. G. Persson, 2012: Outlier problem in evaluating similarity functions in the stable atmospheric boundary layer. Bound.-Layer Meteor., 144, 137-155, doi:10.1007/s10546-012-9714-9.

Högström, U., 1996: Review of some basic characteristics of the atmospheric surface layer. Bound.-Layer Meteor., 78, 215-246.

Holtslag, A. A. M., and A. P. Van Ulden, 1982: Simple estimates of nighttime surface fluxes from routine weather data. Royal Netherlands Meteorological Institute Scientific Rep. 82-4, $11 \mathrm{pp}$.

Howard, L. N., 1961: Note on a paper of John W. Miles. J. Fluid Mech., 10, 509-512.

Jiménez, M., and J. Cuxart, 2005: Large-eddy simulations of the stable boundary layer using the standard Kolmogorov theory: Range of applicability. Bound.-Layer Meteor., 115, 241-261.

King, J. C., and W. M. Connolly, 1997: Validation of the surface energy balance over the Antarctic Ice Sheets in the U.K. Meteorological Office Unified Climate Model. J. Climate, 10, 1273-1287.

Kundu, P. K., and I. M. Cohen, 2008: Fluid Mechanics. 4th ed. Academic Press, 872 pp.

Mahrt, L., 1981: The early evening boundary layer transition. Quart. J. Roy. Meteor. Soc., 107, 329-343.

_ 2011: The near-calm stable boundary layer. Bound.-Layer Meteor., 140, 343-360.

— conditions. Bound.-Layer Meteor., 119, 19-39.

_ J. Sun, W. Blumen, T. Delany, and S. Oncley, 1998: Nocturnal boundary layer regimes. Bound.-Layer Meteor., 88, 255-278.

—, S. Richardson, N. Seaman, and D. Stauffer, 2012: Turbulence in the nocturnal boundary layer with light and variable winds. Quart. J. Roy. Meteor. Soc., 138, 1430-1439, doi:10.1002/qj.1884.

Malhi, Y. S., 1995: The significance of the dual solutions for heat fluxes measured by the temperature fluctuation method in stable conditions. Bound.-Layer Meteor., 74, 389-396.

Mauritsen, T., and G. Svensson, 2007: Observations of stably stratified shear-driven atmospheric turbulence at low and high Richardson numbers. J. Atmos. Sci., 64, 645-655.

McNider, R. T., and R. A. Pielke, 1981: Diurnal boundary layer development over sloping terrain. J. Atmos. Sci., 38, 2198 2212.

—, D. E. England, M. J. Friedman, and X. Shi, 1995: Predictability of the stable atmospheric boundary layer. J. Atmos. Sci., 52, 1602-1614.

Miles, J. W., 1961: On the stability of heterogeneous shear flows. J. Fluid Mech., 10, 496-508.

Moene, A. F., B. J. H. Van de Wiel, and H. J. J. Jonker, 2010: Local similarity profiles from direct numerical simulation. Preprints, 19th Symp. on Boundary Layers and Turbulence, Keystone, CO, Amer. Meteor. Soc., 3A.2. [Available online at https:// ams.confex.com/ams/19Ag19BLT9Urban/techprogram/paper_ 172247.htm.]

Monin, A. S., and A. M. Obukhov, 1954: Basic laws of turbulent mixing in the atmosphere near the ground. Tr. Geofiz. Inst., Akad. Nauk SSSR, 24, 1963-1987.

Nieuwstadt, F. T. M., 1984: The turbulent structure of the stable, nocturnal boundary layer. J. Atmos. Sci., 41, 2202-2216.
_ 2005: Direct numerical simulation of stable channel flow at large stability. Bound.-Layer Meteor., 116, 277-299.

Ohya, Y., D. E. Neff, and R. N. Merony, 1997: Turbulence structure in a stratified boundary layer under stable conditions. Bound.-Layer Meteor., 83, 139-161.

Poulos, G. S., and Coauthors, 2002: CASES-99: A comprehensive investigation of the stable nocturnal boundary layer. Bull. Amer. Meteor. Soc., 83, 555-581.

Revelle, D. O., 1993: Chaos and "bursting" in the planetary boundary layer. J. Atmos. Meteor., 32, 1169-1180.

Schubert, J. F., 1977: Acoustic detection of momentum transfer during the abrupt transition from a laminar to a turbulent atmospheric boundary layer. J. Atmos. Meteor., 16, 1292-1297.

Shapiro, A., and E. Fedorovich, 2008: Coriolis effects in homogeneous and inhomogeneous katabatic flows. Quart. J. Roy. Meteor. Soc., 134, 353-370.

$\longrightarrow$, and - 2010: Analytical description of a nocturnal lowlevel jet. Quart. J. Roy. Meteor. Soc., 136, 1255-1262.

Smedman, A.-S., H. Bergström, and B. Grisogono, 1997: Evolution of stable internal boundary layers over a cold sea. J. Geophys. Res., 102, 1091-1099.

Sorbjan, Z., 2006: Local structure of turbulence in stably stratified boundary layers. J. Atmos. Sci., 63, 1526-1537.

_ 2010: Gradient-based scales and similarity laws in the stable boundary layer. Quart. J. Roy. Meteor. Soc., 136, 1243-1254.

Steeneveld, G. J., B. J. H. van de Wiel, and A. A. M. Holtslag, 2006: Modeling the evolution of the atmospheric boundary layer coupled to the land surface for three contrasting nights in CASES-99. J. Atmos. Sci., 63, 920-935.

Sun, J., and Coauthors, 2003a: Atmospheric disturbances that generate intermittent turbulence in nocturnal boundary layers. Bound.-Layer Meteor., 110, 255-279.

—, S. P. Burns, A. C. Delany, S. P. Oncley, T. W. Horst, and D. H. Lenshow, 2003b: Heat balance in the nocturnal boundary layer during CASES-99. J. Appl. Meteor., 42, 1649-1666.

Svensson, G., and Coauthors, 2011: Evaluation of the diurnal cycle in the atmospheric boundary layer over land as represented by a variety of single-column models: The second GABLS experiment. Bound.-Layer Meteor., 140,177-206.

Taylor, P. A., 1971: A note on the log-linear velocity profile in stable conditions. Quart. J. Roy. Meteor. Soc., 97, 326-329.

Van de Wiel, B. J. H., R. J. Ronda, A. F. Moene, H. A. R. De Bruin, and A. A. M. Holtslag, 2002a: Intermittent turbulence and oscillations in the stable boundary layer. Part I: A bulk model. J. Atmos. Sci., 59, 942-958.

—, A. F. Moene, R. J. Ronda, H. A. R. De Bruin, and A. A. M. Holtslag, 2002b: Intermittent turbulence and oscillations in the stable boundary layer. Part II: A system dynamics approach. J. Atmos. Sci., 59, 2567-2581.

- - O. K. Hartogensis, H. A. R. De Bruin, and A. A. M. Holtslag, 2003: Intermittent turbulence and oscillations in the stable boundary layer. Part III: A classification for observations during CASES99. J. Atmos. Sci., 60, 2509-2522.

,-- G. J. Steeneveld, O. K. Hartogensis, and A. A. M. Holtslag, 2007: Predicting the collapse of turbulence in stably stratified boundary layers. Flow Turbul. Combust., 79, 251274.

W. H. De Ronde, and H. J. J. Jonker, 2008: Local similarity in the stable boundary layer and mixing-length approaches: Consistency of concepts. Bound.-Layer Meteor., 128, 103-116

, H. J. J. Jonker, P. Baas, S. Basu, J. M. M. Donda, S. Sun, and A. A. M. Holtslag, 2012: The minimum wind speed for 
sustainable turbulence in the nocturnal boundary layer. J. Atmos. Sci., 69, 3116-3127.

Wieringa, J., 1989: Shapes of annual frequency distributions of wind speed observed on high meteorological masts. Bound.-Layer Meteor., 47, 85-110.

Zhou, B., and F. K. Chow, 2011: Large-eddy simulation of the stable boundary layer with explicit filtering and reconstruction turbulence modeling. J. Atmos. Sci., 68, 2142-2155.
Zilitinkevich, S. S., T. Elperin, N. Kleeorin, and I. Rogachevskii, 2007: Energy- and flux-budget (EFB) turbulence closure model for stably stratified flows. Part I: Steady state, homogeneous regimes. Bound.-Layer Meteor., 125, 167-191.

,,,---- , I. Esau, T. Mauritsen, and M. W. Miles, 2008: Turbulent energetic in stably stratified geophysical flows: Strong and weak mixing regimes. Quart. J. Roy. Meteor. Soc., 134, 793-799. 